\title{
Korn and Poincaré-Korn inequalities for functions with a small jump set
}

\author{
Filippo Cagnetti ${ }^{1}\left[\right.$ (1) Antonin Chambolle ${ }^{2} \cdot$ Lucia Scardia $^{3}$
}

Received: 25 June 2020 / Revised: 9 April 2021 / Accepted: 13 May 2021 / Published online: 8 June 2021

(c) The Author(s) 2021

\begin{abstract}
In this paper we prove a regularity and rigidity result for displacements in $G S B D^{p}$, for every $p>1$ and any dimension $n \geq 2$. We show that a displacement in $G S B D^{p}$ with a small jump set coincides with a $W^{1, p}$ function, up to a small set whose perimeter and volume are controlled by the size of the jump. This generalises to higher dimension a result of Conti, Focardi and Iurlano. A consequence of this is that such displacements satisfy, up to a small set, Poincaré-Korn and Korn inequalities. As an application, we deduce an approximation result which implies the existence of the approximate gradient for displacements in $G S B D^{p}$.
\end{abstract}

\section{Introduction}

The modelling and analysis of fracture in the linearised elasticity framework relies on a good understanding of the space $B D$ of functions of bounded deformation. These are vector-valued functions $u$ in $L^{1}$, whose symmetric (distributional) gradient $E u$ is a bounded Radon measure. Over the years, the fine properties of functions in $B D$, and in the subspace $S B D$ of special functions of bounded deformation (corresponding to the case where $E u$ has no Cantor part) have been better understood, and the relation between $B D, S B D$ and the space $B V$ of functions of bounded variation has been

Communicated by Y. Giga.

$凶$ Filippo Cagnetti

F.Cagnetti@sussex.ac.uk

Antonin Chambolle

chambolle@ceremade.dauphine.fr

Lucia Scardia

L.Scardia@hw.ac.uk

1 Department of Mathematics, University of Sussex, Brighton, UK

2 CEREMADE, CNRS and Université Paris-Dauphine PSL, Paris, France

3 Department of Mathematics, Heriot-Watt University, Edinburgh, UK 
studied in detail (see e.g., [3,5,17], and [22] for the space of generalised functions of bounded deformation). For a function $u \in S B D(\Omega), E u$ admits the decomposition

$$
E u=e(u) \mathcal{L}^{n}+[u] \odot v_{u} \mathcal{H}^{n-1}\left\llcorner J_{u},\right.
$$

where $e(u)$ is the absolutely continuous part of $E u$ with respect to the Lebesgue measure $\mathcal{L}^{n}, J_{u}$ the jump set of $u$, [u] the jump of $u, v_{u}$ the normal to $J_{u}$ and $[u] \odot v_{u}$ denotes the symmetric tensor product of $u$ and $v_{u}$. The decomposition (1.1) has a clear physical meaning: $e(u)$ represents the elastic part of the strain, and $J_{u}$ the crack set. It is therefore natural that a model of (brittle) fracture, in the linearised setting, would involve an energy of the type

$$
\int_{\Omega}|e(u)|^{2} d x+\mathcal{H}^{n-1}\left(J_{u}\right),
$$

called the Griffith's energy, of which the Mumford-Shah energy in $S B V$ is the scalar counterpart. The energy (1.2) is in fact well defined in the larger space $G S B D(\Omega)$ of generalised special functions of bounded deformation, which has been introduced by Dal Maso in [22], and is essentially designed to contain all the displacements for which the energy is finite (see Sect. 2 for the definition). Moreover, GSBD is the natural space for (1.2), where one can prove compactness and existence of minimisers under physical assumptions (see, e.g., $[12,13,15])$.

A key difficulty posed by the energy (1.2), compared to scalar models based on functions of bounded variation, is the lack of control on the skew-symmetric part $\left(D u-D u^{T}\right) / 2$ of the distributional gradient of $u$. The classical tool providing a relation between the full gradient and its symmetric part is the Korn inequality.

In this paper we prove Korn and Poincaré-Korn inequalities in $G S B D^{p}(\Omega)$, the space of functions $u \in G S B D(\Omega)$ for which $e(u) \in L^{p}(\Omega)$ and $\mathcal{H}^{n-1}\left(J_{u}\right)<+\infty$, for every dimension $n \geq 2$ and any $p>1$. More precisely, we have the following (see Theorem 4.5).

Theorem 1.1 Let $n \in \mathbb{N}$ with $n \geq 2, p \in(1, \infty)$, and let $\Omega \subset \mathbb{R}^{n}$ be a bounded, open and connected Lipschitz set. Then, there exists $c=c(n, p, \Omega)>0$ with the following property. For any $u \in G S B D^{p}(\Omega)$, there exist a set of finite perimeter $\omega \subset \Omega$ with $\mathcal{H}^{n-1}\left(\partial^{*} \omega\right) \leq c \mathcal{H}^{n-1}\left(J_{u}\right)$, and an infinitesimal rigid motion a (namely an affine function $a$, with $e(a)=0)$, such that

$$
\int_{\Omega \backslash \omega}|\nabla u-\nabla a|^{p} d x \leq c(n, p, \Omega) \int_{\Omega}|e(u)|^{p} d x .
$$

Moreover, there exists $c=c(n, p, q, \Omega)>0$ such that

$$
\|u-a\|_{L^{q}(\Omega \backslash \omega)} \leq c(n, p, q, \Omega)\|e(u)\|_{L^{p}(\Omega)},
$$

with $q \leq p^{*}$ if $p<n, q<\infty$ if $p=n$, and $q \leq \infty$ for $p>n$. 
Clearly, the volume of $\omega$ is also controlled by $\mathcal{H}^{n-1}\left(J_{u}\right)$, thanks to the isoperimetric inequality, see Remark 3.4 below.

This result is the generalisation, in dimension $n \geq 2$, of the two-dimensional result in [16] (see also [27]). Theorem 1.1 ensures that $e(u)$ controls $u-a$ and its approximate gradient outside an exceptional set, and not in the whole set $\Omega$. This is in contrast with the classical Korn and Poincaré-Korn inequalities for functions $u \in W^{1, p}\left(\Omega ; \mathbb{R}^{n}\right)$, with $p>1$, which state that there exists an infinitesimal rigid motion $a$ such that

$$
\|D u-D a\|_{L^{p}(\Omega)} \leq c(n, p, \Omega)\|E u\|_{L^{p}(\Omega)},
$$

and that, thanks to the Poincaré inequality and Sobolev embeddings,

$$
\|u-a\|_{L^{q}(\Omega)} \leq c(n, p, q, \Omega)\|E u\|_{L^{p}(\Omega)},
$$

where $q$ depends on $n$ and $p$ (and $q=p^{*}$ for $p<n$ ).

Results like (1.5) and (1.6) are clearly out of reach in $(G) S B D$, even for functions $u$ with a small jump set. This is due to the possible presence of small regions of $\Omega$ that can be completely (or almost completely) disconnected from the domain, and where $u$ would not necessarily be close to the infinitesimal rigid motion that achieves the smallest distance from $u$ in the majority of the domain. Hence, in general, for a function $u \in(G) S B D(\Omega), e(u)$ cannot control $u-a$ or its approximate gradient in the whole domain $\Omega$, and a result like Theorem 1.1 is the best possible.

The Korn and Poincaré-Korn inequalities in Theorem 1.1 are a corollary of the result below (see Theorem 4.1 and Remark 4.3), which is the main result of this paper.

Theorem 1.2 Let $n \in \mathbb{N}$ with $n \geq 2, p \in(1, \infty)$, and let $\Omega \subset \mathbb{R}^{n}$ be a bounded and open Lipschitz set. Then, there exists $c=c(n, p, \Omega)>0$ with the following property. For any $u \in G S B D^{p}(\Omega)$, there exists a set of finite perimeter $\omega \subset \Omega$ with $\mathcal{H}^{n-1}\left(\partial^{*} \omega\right) \leq c \mathcal{H}^{n-1}\left(J_{u}\right)$ and $v \in W^{1, p}\left(\Omega ; \mathbb{R}^{n}\right)$, such that $u=v$ in $\Omega \backslash \omega$ and $\int_{\Omega}|e(v)|^{p} d x \leq c \int_{\Omega}|e(u)|^{p} d x$. If in addition $u$ is bounded, then $\|v\|_{L^{\infty}(\Omega)} \leq$ $\|u\|_{L^{\infty}(\Omega)}$.

In Theorem 1.2, we prove 'almost' Sobolev regularity for functions in $G S B D^{p}$. More precisely we show that, given a function $u \in G S B D^{p}(\Omega)$, we can replace it with a function $v \in W^{1, p}\left(\Omega ; \mathbb{R}^{n}\right)$ outside an exceptional set $\omega \subset \Omega$, whose perimeter is controlled by $\mathcal{H}^{n-1}\left(J_{u}\right)$. Moreover, $u$ and $v$ have a comparable Griffith's energy in the whole of $\Omega$. We observe that the conclusion of Theorem 1.2 is non-trivial only when the measure of the jump set $J_{u}$ is 'small' as else one could take $\omega=\Omega$ and $v=0$ (see also Remark 4.2). The proof of Theorem 1.2 is done by regularising $u$ at several scales, by means of the auxiliary results Lemma 3.1 and Theorem 3.2.

We now illustrate the idea of the proof. As a first step, we cover the domain $\Omega$ with a family of disjoint cubes $q$ whose size reduces towards the boundary. The cubes in the partition are then classified into 'good' and 'bad', depending on whether the amount of $J_{u}$ they contain is smaller or larger than a given threshold. The construction is done so that all the cubes in the covering of $\Omega$ are 'good', up to a small neighbourhood of $\partial \Omega$. In this neighbourhood, the 'bad' cubes are cut away from the domain by connecting them 
to $\partial \Omega$ by means of truncated cones. In this way what remains is still a Lipschitz set (with the same Lipschitz constant as $\Omega$ ). Moreover, in each 'bad' cube, by definition, the perimeter of the cone is comparable to the perimeter of the cube, and hence is bounded by the measure of the jump set of $u$ in it.

Hence it is sufficient to deal with good cubes. For each of the good cubes $q$ we apply the auxiliary regularity result Theorem 3.2. This ensures that, given a function $\tilde{u} \in G S B D^{p}(q)$, we can wipe out its jump set $J_{\tilde{u}}$ away from the boundary of $q$, up to a small expense in terms of the Griffith's energy, provided $\mathcal{H}^{n-1}\left(J_{\tilde{u}}\right)$ is sufficiently smaller than the perimeter of $q$. This 'smallness' condition is exactly what enters in the definition of 'good' cubes. Applying Theorem 3.2 to $\tilde{u}:=u_{\mid q}$ in every 'good' cube $q$, we obtain a Sobolev regularisation $\tilde{v}_{q}$ of $u_{\mid q}$ and an exceptional set $\tilde{\omega}_{q}$ with controlled perimeter, such that $\tilde{v}_{q}=u$ outside $\tilde{\omega}_{q}$. The function $v$ in the statement of Theorem 1.2 is then obtained by patching together the functions $\tilde{v}_{q}$ on all the good cubes. The set $\omega$ where $v$ needs not coincide with $u$, is then defined as the union of the exceptional sets $\tilde{\omega}_{q}$ of the good cubes, together with the truncated cones relative to the bad cubes.

To conclude, we sketch the proof of Theorem 3.2, which is strongly inspired by its two-dimensional version [16], by Conti, Focardi and Iurlano, and involves an iterative regularisation procedure. Starting with $w_{0}=\tilde{u}$, we construct a sequence $\left(w_{k}\right)$, where $w_{k+1}$ is obtained by covering a large part of $J_{w_{k}}$ with a family of disjoint balls, and by replacing $w_{k}$ in each ball of the covering with a smoother function, provided by Lemma 3.1. The pointwise limit $\tilde{v}$ of the sequence $\left(w_{k}\right)$ has Sobolev regularity in a smaller ball, and satisfies $\tilde{u}=\tilde{v}$ outside an exceptional set $\tilde{\omega}$, which is defined as the union of the coverings of each step.

\subsection{Comparison with previous results}

The foundations of the function spaces $S B D$ and $G S B D$ were laid down in the papers $[3,5]$, and [22]. Several research avenues have stemmed from them: the derivation of regularity properties for functions in $(G) S B D^{p}$, and in particular of minimisers of the Griffith's energy, in the spirit of the celebrated result [23] by De Giorgi, Carriero and Leaci for the Mumford-Shah energy (see [4,10,12,13,15,17]); of Korn and PoincaréKorn inequalities with various degrees of generality ([9,26-28]); of approximation and density results $([7,11,18-20,29])$; of integral representation for functionals in (G) $S B D^{p}[16]$.

Our results are in between two of these avenues: we prove Sobolev regularity for functions in $G S B D^{p}$, for every $p>1$ and in every dimension $n \geq 2$, outside an exceptional set (see Theorem 1.2) and, as a direct corollary, we obtain a Korn inequality, and a Poincaré-Korn inequality with sharp exponent (see Theorem 1.1), again outside an exceptional set.

Our work has a number of points of contact with previous results, but also a number of differences. In [9] the authors prove a Poincaré-Korn inequality like (1.4) for every $n \geq 2$ and every $p \geq 1$ by means of a slicing argument. Unlike our case, however, they obtain (1.4) with $q=p\left(1^{*}\right)$, rather than $q=p^{*}$ (which is optimal only for $p=1$ ), and no estimate for the gradient of $u$ is provided. Moreover, the exceptional set $\omega$ is 
controlled by the jump set of $J_{u}$ only in volume, while we also control its perimeter. A Poincaré-Korn inequality like (1.4) is proved also in [26], for $n=2$ and $p=2$, with an exceptional set $\omega$ whose structure is very simple, and can be related to the measure of $J_{u}$. This objective is further pursued in [28], where the author proves a PoincaréKorn inequality in $G S B D^{2}$, up to an exceptional set with both perimeter and area bounded by (powers of) the measure of $J_{u}$, for $n \geq 2$. The $L^{2}$-norm of $e(u)$, however, only controls the distance of $u$ from a rigid motion in the weaker norm $q=2\left(1^{*}\right)$; additionally, one can obtain an $L^{\infty}$ bound for such a distance, but the $L^{2}$-norm of $e(u)$ has to be weighted with a negative power of the measure of $J_{u}$.

The first proofs of a Korn inequality like (1.3), in the $(G) S B D$ context, are due to [27] and [16]. In [27] the proof is done in dimension $n=2$ and for $p=2$. Moreover, the distance of $\nabla u$ from a skew-symmetric matrix is estimated in a lower $L^{q}$-norm, with $q \in[1,2)$. On the other hand, the exceptional set is estimated, both in perimeter and in area, with the measure of $J_{u}$, and the integrability of $u$ is improved to the sharp exponent, with consequent improvement of the Poincaré-Korn inequality. The two-dimensionality of the result is due to an approximation step, done in [26], that is only proved in the planar setting. Also the result in [16] is only proved for $n=2$, and again this is due to a 'regularisation' step being done by means of a two-dimensional construction. Their approach, like ours, is based on first proving Sobolev regularity outside an exceptional set, and then deducing Korn and Poincaré-Korn inequalities as direct corollaries. Also in [16], like in our result, the exceptional set is bounded in perimeter in terms of $J_{u}$, and the Poincaré-Korn inequality is proved with the sharp exponent for every $p>1$.

In conclusion, our contribution is two-fold. On the one hand our result lifts the restriction to dimension $n=2$ of the regularisation step from $G S B D^{p}$ to $W^{1, p}$, up to an exceptional set, which is now valid for every $n \geq 2$ and every $p>1$. In addition, the exceptional set we provide is bounded both in perimeter and in area with the measure of the jump set of the function. As a consequence, we can deduce the Korn and Poincaré-Korn inequalities up to the sharp exponent for every $n \geq 2$ and $p>1$, since the regularisation step is not reliant on a planar construction.

\subsection{Conclusion and perspectives}

The main result in this work, asserting the 'almost' Sobolev regularity of $G S B D^{p}$ functions with a small jump set, has some nontrivial consequences which are of independent interest, and which we present here. First of all, we obtain a Korn and a Poincaré-Korn inequality with sharp exponents outside an exceptional set, which is controlled in perimeter and volume by the jump set of the function (Theorem 4.5). We also prove an approximation result (Theorem 5.1) in the spirit of [11, Theorem 3.1]. Theorem 5.1 implies, in particular, the existence of the approximate gradient $\nabla u$ for functions in $G S B D^{p}$ (Corollary 5.2). Note that the existence of $\nabla u$ for functions in $G S B D^{2}$ had already been obtained in [28], as a consequence of the embedding $G S B D^{2}(\Omega) \subset(G B V(\Omega))^{n}$ (see [28, Theorem 2.9]), for $n \geq 2$.

In analogy with [16], our result has been recently used to obtain an integral representation result for functionals in $G S B D^{p}$ in higher dimension, see [21]. 
Moreover, the 'almost' Sobolev regularity of $G S B D^{p}$-functions with a small jump set, Theorem 1.2, is one of the main ingredients of the extension result [6]; additionally, the Korn-Poincaré inequality (Theorem 4.5) and the approximation result (Theorem 5.1) have been used in [14] to prove compactness and lower-semicontinuity for nonhomogeneous Griffith-like energies.

\section{Notation}

We introduce now some notation that will be used throughout the paper.

(a) $\mathcal{L}^{n}$ denotes the Lebesgue measure on $\mathbb{R}^{n}$ and $\mathcal{H}^{n-1}$ the $(n-1)$-dimensional Hausdorff measure on $\mathbb{R}^{n}$.

(b) $e_{1}, \ldots, e_{n}$ is the canonical basis of $\mathbb{R}^{n} ;|\cdot|$ denotes the absolute value in $\mathbb{R}$ or the Euclidean norm in $\mathbb{R}^{n}$, depending on the context, and $\cdot$ denotes the Euclidean scalar product. We set $\mathbb{S}^{n-1}:=\left\{x \in \mathbb{R}^{n}:|x|=1\right\}$. We denote with $\mathbb{R}_{\text {sym }}^{n \times n}$ the set of symmetric $n \times n$ matrices.

(c) For $x \in \mathbb{R}^{n}$ and $\rho>0$ we define the ball:

$$
B_{\rho}(x):=\left\{y \in \mathbb{R}^{n}:|y-x|<\rho\right\} .
$$

(d) For $x \in \mathbb{R}^{n}$, e $\in \mathbb{S}^{n-1}$, and $\rho>0$, we define the cylinder:

$$
C(x, \mathrm{e}, h, \rho):=\left\{y \in \mathbb{R}^{n}:|(y-x) \cdot \mathrm{e}|<h,|(y-x)-((y-x) \cdot \mathrm{e}) \mathrm{e}|<\rho\right\} .
$$

(e) For $y \in \mathbb{R}^{n}$ and $\xi \in \mathbb{S}^{n-1}$, we set:

$$
\Pi_{y}^{\xi}:=\left\{x \in \mathbb{R}^{n}:(x-y) \cdot \xi=0\right\},
$$

and use the shorthand $\Pi^{\xi}=\Pi_{0}^{\xi}$.

(f) For $a, b \in \mathbb{R}^{n}$, we denote with $a \otimes b \in \mathbb{R}^{n \times n}$ the tensor product of $a$ and $b$, namely the matrix with $(a \otimes b)_{i j}=a_{i} b_{j}$ for every $i, j=1, \ldots, n$. Moreover, we denote the symmetrised tensor product as $a \odot b:=(a \otimes b+b \otimes a) / 2 \in \mathbb{R}_{\mathrm{sym}}^{n \times n}$.

(g) $\mathcal{R}$ is the set of infinitesimal rigid motions in $\mathbb{R}^{n}$, namely $a \in \mathcal{R}$ if and only if $a: x \mapsto A x+b$, with $A \in \mathbb{R}^{n \times n}$ skew-symmetric, and $b \in \mathbb{R}^{n}$.

(h) For every $t \in[0,1]$ and every $\mathcal{L}^{n}$-measurable set $E \subset \mathbb{R}^{n}$ we denote with $E^{(t)}$ the set of all points where $E$ has density $t$, namely

$$
E^{(t)}:=\left\{x \in \mathbb{R}^{n}: \lim _{\rho \downarrow 0} \frac{\mathcal{L}^{n}\left(E \cap B_{\rho}(x)\right)}{\mathcal{L}^{n}\left(B_{\rho}(x)\right)}=t\right\} .
$$

(i) An $\mathcal{L}^{n}$-measurable and bounded set $E \subset \mathbb{R}^{n}$ is a set of finite perimeter if its characteristic function $\chi_{E}$ is a function of bounded variation. The reduced boundary of $E$, denoted with $\partial^{*} E$ is the set of points $x \in \operatorname{supp}\left|D \chi_{E}\right|$ where a generalised normal $v_{E}$ is defined. 
(j) For $\Omega \subset \mathbb{R}^{n}$ measurable, $\mathcal{M}_{b}\left(\Omega ; \mathbb{R}^{m}\right)$ denotes the space of bounded Radon measures with values in $\mathbb{R}^{m}$, for $m \geq 1$. Moreover, for $m=1$, we denote with $\mathcal{M}_{b}^{+}(\Omega)$ the sub-class of positive measures.

(k) For $k \in \mathbb{N}, \gamma_{k} \in \mathbb{R}$ denotes the $k$-dimensional Lebesgue measure of the unit ball in $\mathbb{R}^{k}$. With this notation, we have $\mathcal{H}^{n-1}\left(\mathbb{S}^{n-1}\right)=n \gamma_{n}$.

Let $\Omega \subset \mathbb{R}^{n}$ be an open set. We now introduce the functional spaces we will work with in this paper. We first recall the definition of the space $G B D$ of generalised functions with bounded deformation, which is due to Dal Maso [22] and relies on slicing. Given an $\mathcal{L}^{n}$-measurable function $u: \Omega \rightarrow \mathbb{R}^{n}$, we say that $u \in G B D(\Omega)$ if there exists $\lambda_{u} \in \mathcal{M}_{b}^{+}(\Omega)$ such that the following is true for every $\xi \in \mathbb{S}^{n-1}$ :

- For every $\tau \in C^{1}(\mathbb{R})$ with $-\frac{1}{2} \leq \tau \leq \frac{1}{2}$ and $0 \leq \tau^{\prime} \leq 1$

$$
D_{\xi}(\tau(u \cdot \xi))=D(\tau(u \cdot \xi)) \cdot \xi \in \mathcal{M}_{b}(\Omega)
$$

- For every Borel set $B \subset \Omega$

$$
\left|D_{\xi}(\tau(u \cdot \xi))\right|(B) \leq \lambda_{u}(B)
$$

We say that $u \in G S B D(\Omega)$ if in addition $\hat{u}_{y}^{\xi}(t) \in S B V_{\text {loc }}\left(\Omega_{y}^{\xi}\right)$ for every $\xi \in \mathbb{S}^{n-1}$ and for $\mathcal{H}^{n-1}$-a.e. $y \in \Pi^{\xi}$, where $\Omega_{y}^{\xi}:=\{t \in \mathbb{R}: y+t \xi \in \Omega\}$ and, for $t \in \Omega_{y}^{\xi}$, $\hat{u}_{y}^{\xi}(t):=u(y+t \xi) \cdot \xi$ denotes the slice of $u$ in the direction $\xi$. In [22] it is shown that, given a function $u \in G S B D(\Omega)$, one can define an 'approximate symmetrised gradient' $e(u) \in L^{1}\left(\Omega ; \mathbb{R}_{\text {sym }}^{n \times n}\right)$ as well as an $\left(\mathcal{H}^{n-1}, n-1\right)$-countably rectifiable jump set $J_{u}$, which both coincide with the standard definitions [3] if $u \in B D(\Omega)$. Finally, we recall the definition of the space $G S B D^{p}$, namely

$$
G S B D^{p}(\Omega):=\left\{u \in G S B D(\Omega): e(u) \in L^{p}\left(\Omega ; \mathbb{R}_{\mathrm{sym}}^{n \times n}\right), \mathcal{H}^{n-1}\left(J_{u}\right)<+\infty\right\} .
$$

\section{How to wipe out small jump sets}

The following Lemma is a variant of [10, Theorem 3], which can be proved by adapting the arguments to the case of a ball. This result ensures that a $G S B D^{p}$-function with a small jump set in the unit ball can be regularised away from the boundary, up to a small cost in Griffith's energy.

Lemma 3.1 Let $n \in \mathbb{N}$ with $n \geq 2$, and let $p \in(1, \infty)$. There exist $\bar{\delta}, c$, s positive constants, depending only on $n$ and $p$, with the following property. For every $u \in$ $G S B D^{p}\left(B_{1}\right)$ with $\delta:=\mathcal{H}^{n-1}\left(J_{u}\right)^{1 / n} \leq \bar{\delta}$, there exists $\tilde{u} \in G S B D^{p}\left(B_{1}\right)$ and $R \in$ $(1-\sqrt{\delta}, 1)$ such that

(1) $\tilde{u} \in C^{\infty}\left(B_{1-\sqrt{\delta}}\right), \tilde{u}=u$ in $B_{1} \backslash B_{R}$, and $\mathcal{H}^{n-1}\left(J_{u} \cap \partial B_{R}\right)=\mathcal{H}^{n-1}\left(J_{\tilde{u}} \cap \partial B_{R}\right)=0$;

(2) $\mathcal{H}^{n-1}\left(J_{\tilde{u}} \backslash J_{u}\right) \leq c \sqrt{\delta} \mathcal{H}^{n-1}\left(J_{u} \cap\left(B_{1} \backslash B_{1-\sqrt{\delta}}\right)\right)$; 
(3) it holds

$$
\int_{B_{1}}|e(\tilde{u})|^{p} d x \leq\left(1+c \delta^{s}\right) \int_{B_{1}}|e(u)|^{p} d x
$$

(4) if in addition $u$ is bounded, then one can ensure $\|\tilde{u}\|_{L^{\infty}\left(B_{1}\right)} \leq\|u\|_{L^{\infty}\left(B_{1}\right)}$.

The last point follows from Remark 6 and Lemma A.1 in [8], which can be used when building the function $\tilde{u}$ in the construction of [10, Theorem 3].

The following theorem is an extension in dimension $n \geq 2$ of a planar result of Conti, Focardi and Iurlano [16]. Our proof is strongly inspired by theirs and involves an iterative regularisation procedure and a covering argument. Essentially, it shows that a $G S B D^{p}$-function with a small jump set coincides, outside a small neighbourhood of the jump set, with a function that has Sobolev regularity away from the boundary. Moreover, the energy of the regularised function can be made arbitrarily close to the energy of the original function.

Theorem 3.2 Let $n \in \mathbb{N}$ with $n \geq 2$, and let $p \in(1, \infty)$. Given $\varepsilon>0$ and $\sigma \in(0,1)$, there exist $C=C(n, p, \varepsilon)>0$ and $\tau=\tau(n, p, \varepsilon, \sigma)>0$ with the following property. For every $\rho>0$ and $u \in G S B D^{p}\left(B_{\rho}\right)$ with $\mathcal{H}^{n-1}\left(J_{u}\right) \leq \tau \rho^{n-1}$, there exists $w \in G S B D^{p}\left(B_{\rho}\right)$ and a set of finite perimeter $\omega \subset B_{\rho}$, such that $w=u$ in $B_{\rho} \backslash \omega, \mathcal{H}^{n-1}\left(\partial^{*} \omega\right) \leq C \mathcal{H}^{n-1}\left(J_{u}\right), w \in W^{1, p}\left(B_{(1-\sigma) \rho} ; \mathbb{R}^{n}\right)$, and

$$
\int_{B_{\rho}}|e(w)|^{p} d x \leq(1+\varepsilon) \int_{B_{\rho}}|e(u)|^{p} d x, \quad \mathcal{H}^{n-1}\left(J_{w}\right) \leq \mathcal{H}^{n-1}\left(J_{u}\right) .
$$

Moreover if $u$ is bounded, one can ensure $\|w\|_{L^{\infty}\left(B_{\rho}\right)} \leq\|u\|_{L^{\infty}\left(B_{\rho}\right)}$.

Remark 3.3 A careful inspection of the proof shows that

$$
\lim _{\sigma \rightarrow 0^{+}} \tau(n, p, \varepsilon, \sigma)=\lim _{\varepsilon \rightarrow 0^{+}} \tau(n, p, \varepsilon, \sigma)=0,
$$

and

$$
\lim _{\varepsilon \rightarrow 0^{+}} C(n, p, \varepsilon)=+\infty \text {. }
$$

Remark 3.4 Note that also the volume of $\omega$ is controlled by the measure of the jump set $J_{u}$ of $u$. Indeed, the isoperimetric inequality ensures that $\left(\mathcal{L}^{n}(\omega)\right)^{(n-1) / n} \leq$ $C \mathcal{H}^{n-1}\left(J_{u}\right)$ (possibly changing the constant $C$ ). In addition, since $\omega \subset B_{\rho}$, we have $\left(\mathcal{L}^{n}(\omega)\right)^{1 / n} \leq \gamma_{n}^{1 / n} \rho$. Multiplying these two inequalities we obtain that $\mathcal{L}^{n}(\omega) \leq$ $C \rho \mathcal{H}^{n-1}\left(J_{u}\right)$.

Proof Choose $\varepsilon>0$ and $\sigma \in(0,1)$, and let $\rho>0$ and $u \in G S B D^{p}\left(B_{\rho}\right)$. We start by assuming that

$$
\mathcal{H}^{n-1}\left(J_{u}\right) \leq \tau \rho^{n-1},
$$


for a $\tau>0$ to be determined later (see (3.27)).

The function $w$ in the thesis of the theorem will be obtained as the pointwise limit of a sequence $\left(w_{k}\right)_{k \geq 0}$, constructed iteratively starting from $w_{0}=u$, and by progressively "wiping out" parts of the jump of $u$, at the expense of a controlled increase of the $L^{p}$ norm of the approximate symmetric gradient. We split the proof into several steps.

Step 1: Iterative construction of $\left(w_{k}\right)_{k \geq 0}$. We will now build a sequence of functions $\left(w_{k}\right)_{k \geq 0} \subset G S B D^{p}\left(B_{\rho}\right)$ by induction.

Step 1.1: Base case. Let $\bar{\delta}=\bar{\delta}(n, p)$ be the constant given by Lemma 3.1. By possibly reducing its value, we assume in addition that $\gamma_{n-1}>\bar{\delta}^{n}$ (see notation (k) in Section 2). Let also $\alpha=\alpha(n, p, \varepsilon) \in(0,1)$ be a constant to be determined later (see (3.28)). We set $w_{0}:=u, \eta_{0}:=(\alpha \bar{\delta})^{n}, \rho_{0}:=\rho$ and

$$
s_{0}:=\frac{1}{\rho}\left(\frac{\mathcal{H}^{n-1}\left(J_{u}\right)}{\eta_{0}}\right)^{\frac{1}{n-1}}
$$

Note that by assumption $s_{0} \leq\left(\tau / \eta_{0}\right)^{1 /(n-1)}$. In order for the iteration to converge, we will need $s_{0}$ to be sufficiently small, hence the $\tau$ in the statement. We also observe that, by the definition of $s_{0}$, we have

$$
\mathcal{H}^{n-1}\left(J_{w_{0}} \cap B_{\rho_{0}}\right)=\mathcal{H}^{n-1}\left(J_{u} \cap B_{\rho}\right)=\eta_{0}\left(\rho_{0} s_{0}\right)^{n-1} .
$$

Step 1.2: Induction step. Let $k \geq 0$, and suppose we are given $w_{k} \in G S B D^{p}\left(B_{\rho}\right)$, $s_{k} \in(0,1), \rho_{k} \leq \rho$ and $\eta_{k} \leq \bar{\delta}^{n}$ which satisfy

$$
\mathcal{H}^{n-1}\left(J_{w_{k}} \cap B_{\rho_{k}}\right) \leq \eta_{k}\left(s_{k} \rho_{k}\right)^{n-1}
$$

as it is the case for $k=0$. We will build $w_{k+1}, \eta_{k+1}, s_{k+1}$ and $\rho_{k+1}$ (explicitly given at the end of the step) such that (3.3) is satisfied for $k+1$. We will divide the proof of the induction step into further substeps.

Our strategy is the following. We construct a function $w_{k+1}$ whose jump set is (in measure) not larger than the one of the function $w_{k}$. To do so, we cover a large part of $J_{w_{k}}$ in the smaller ball $B_{\left(1-s_{k}\right) \rho_{k}}$ (subsequently defined as $B_{\rho_{k+1}}$ ) with a family of disjoint balls, and we wipe out a significant part of the jump set of $w_{k}$ in each ball of the covering.

Step 1.2a: Construction of the covering. We claim that for $\mathcal{H}^{n-1}$-a.e. $x \in J_{w_{k}} \cap$ $B_{\left(1-s_{k}\right) \rho_{k}}$ there exists $r_{x} \in\left(0, s_{k} \rho_{k}\right]$ such that

$$
\left\{\begin{array}{l}
\mathcal{H}^{n-1}\left(J_{w_{k}} \cap B_{r_{x}}(x)\right)=\eta_{k} r_{x}^{n-1} \\
\mathcal{H}^{n-1}\left(J_{w_{k}} \cap B_{r}(x)\right) \geq \eta_{k} r^{n-1} \quad \text { for } r \leq r_{x}
\end{array}\right.
$$

Indeed, if $x$ is a point of rectifiability of $J_{w_{k}} \cap B_{\left(1-s_{k}\right) \rho_{k}}$ and we define

$$
\phi(r):=\frac{\mathcal{H}^{n-1}\left(J_{w_{k}} \cap B_{r}(x)\right)}{r^{n-1}}, \quad r \in\left(0, s_{k} \rho_{k}\right],
$$


then we have $\lim _{r \rightarrow 0^{+}} \phi(r)=\gamma_{n-1}>\eta_{k}$ (since $\left.\gamma_{n-1}>\bar{\delta}^{n}\right)$. Moreover, since $B_{s_{k} \rho_{k}}(x) \subset B_{\rho_{k}}$, from (3.3) it follows that $\phi\left(s_{k} \rho_{k}\right) \leq \eta_{k}$. Therefore, we have that $r_{x}:=\inf \left\{r \in\left(0, s_{k} \rho_{k}\right): \phi(r) \leq \eta_{k}\right\}>0$. As $\phi$ is lower semicontinuous one has $\phi\left(r_{x}\right) \leq \eta_{k}$, and as it is left-continuous, one has $\phi\left(r_{x}\right) \geq \eta_{k}$. This shows (3.4). By construction, observe also that

$$
\mathcal{H}^{n-1}\left(J_{w_{k}} \cap \partial B_{r_{x}}(x)\right)=0 .
$$

By the Besicovitch Covering Theorem (see, for instance, [2, Theorem 2.17]) there exists a positive integer $\xi(n)$, depending only on $n$, with the following property: There exist $\xi(n)$ countable families of such closed balls $\left(\bar{B}_{r_{x_{i}^{\ell}}}\left(x_{i}^{\ell}\right)\right), i \geq 1$ and $\ell=1, \ldots, \xi(n)$, with $\bar{B}_{r_{x_{i}^{\ell}}}\left(x_{i}^{\ell}\right) \cap \bar{B}_{r_{x_{j}^{\ell}}}\left(x_{j}^{\ell}\right)=\emptyset$ for $i \neq j$, and such that

$$
\mathcal{H}^{n-1}\left(\left(J_{w_{k}} \cap B_{\left(1-s_{k}\right) \rho_{k}}\right) \backslash\left(\bigcup_{\ell=1}^{\xi(n)} \bigcup_{i \geq 1} \bar{B}_{r_{x_{i}^{\ell}}}\left(x_{i}^{\ell}\right)\right)\right)=0
$$

Let us choose $\bar{\ell} \in\{1, \ldots, \xi(n)\}$ such that

$$
\mathcal{H}^{n-1}\left(\left(J_{w_{k}} \cap B_{\left(1-s_{k}\right) \rho_{k}}\right) \cap\left(\bigcup_{i \geq 1} \bar{B}_{r_{x_{i}^{\bar{\ell}}}}\left(x_{i}^{\bar{\ell}}\right)\right)\right)
$$

is maximal. Then one has

$$
\begin{aligned}
\sum_{i \geq 1} \mathcal{H}^{n-1}\left(J_{w_{k}} \cap \bar{B}_{r_{x_{i}^{\bar{\ell}}}}\left(x_{i}^{\bar{\ell}}\right)\right) & \geq \mathcal{H}^{n-1}\left(\left(J_{w_{k}} \cap B_{\left(1-s_{k}\right) \rho_{k}}\right) \cap\left(\bigcup_{i \geq 1} \bar{B}_{r_{x_{i}^{\bar{\ell}}}}\left(x_{i}^{\bar{\ell}}\right)\right)\right) \\
& \geq \frac{1}{\xi(n)} \mathcal{H}^{n-1}\left(J_{w_{k}} \cap B_{\left(1-s_{k}\right) \rho_{k}}\right) .
\end{aligned}
$$

In what follows we denote, to simplify, $B_{i}:=B_{r_{x_{i}^{\bar{\ell}}}}\left(x_{i}^{\bar{\ell}}\right), x_{i}:=x_{i}^{\bar{\ell}}, r_{i}:=r_{x_{i}^{\bar{\ell}}}$.

Step 1.2b: Definition of $w_{k+1}$. We define $w_{k+1}$ in two different ways, depending on whether the amount of the jump set of $w_{k}$ in the annulus $B_{\rho_{k}} \backslash B_{\left(1-s_{k}\right) \rho_{k}}$ is large or not. We first let

$$
\theta:=\frac{2 \xi(n)}{1+2 \xi(n)} \in\left(\frac{2}{3}, 1\right)
$$

In the case

$$
\mathcal{H}^{n-1}\left(J_{w_{k}} \cap B_{\left(1-s_{k}\right) \rho_{k}}\right) \leq \theta \mathcal{H}^{n-1}\left(J_{w_{k}} \cap B_{\rho_{k}}\right)
$$

we let $w_{k+1}:=w_{k}$. If instead we have the reverse inequality in (3.8), and consequently

$$
\mathcal{H}^{n-1}\left(J_{w_{k}} \cap\left(B_{\rho_{k}} \backslash B_{\left(1-s_{k}\right) \rho_{k}}\right)\right) \leq(1-\theta) \mathcal{H}^{n-1}\left(J_{w_{k}} \cap B_{\rho_{k}}\right),
$$


we then define $w_{k+1}$ as

$$
w_{k+1}(x):= \begin{cases}w_{k}(x) & \text { if } x \in B_{\rho} \backslash\left(\bigcup_{i \geq 1} \bar{B}_{i}\right), \\ \widetilde{w}_{k, i}(x) & \text { if } x \in B_{i} \text { for some } i \in \mathbb{N}\end{cases}
$$

where $\widetilde{w}_{k, i} \in G S B D^{p}\left(B_{i}\right)$ denotes the function obtained by applying Lemma 3.1, after suitable translation and rescaling, to the restriction of $w_{k}$ in each ball $B_{i}$ for every $i \geq 1$. Note that in this case the value of $\delta$, by definition of the balls $B_{i}$ (namely by (3.4)), is given by $\eta_{k}^{1 / n}$, and $\eta_{k}^{1 / n} \leq \bar{\delta}$ by the assumption of the induction step.

Step 1.2c: Proof of the induction step. In case (3.8) is satisfied, we have $w_{k+1}=w_{k} \in$ $G S B D^{p}\left(B_{\rho}\right)$, so that

$$
\mathcal{H}^{n-1}\left(J_{w_{k+1}}\right)=\mathcal{H}^{n-1}\left(J_{w_{k}}\right)
$$

and, by using (3.8) and (3.3),

$$
\left.\mathcal{H}^{n-1}\left(J_{w_{k+1}} \cap B_{\left(1-s_{k}\right.}\right) \rho_{k}\right) \leq \theta \eta_{k}\left(s_{k} \rho_{k}\right)^{n-1} .
$$

We now assume that (3.9) holds. By Property (1) of Lemma 3.1 we have $w_{k+1} \in$ $G S B D^{p}\left(B_{\rho}\right)$. Moreover, let $R_{k, i} \in\left(1-\eta_{k}^{1 /(2 n)}, 1\right)$ be the radius given by Lemma 3.1 and corresponding to $\widetilde{w}_{k, i}$. Setting $B_{i}^{\prime}:=B_{\left(1-\eta_{k}^{1 /(2 n)}\right) r_{i}}\left(x_{i}\right)$ and $B_{i}^{\prime \prime}:=B_{R_{k, i} r_{i}}\left(x_{i}\right)$, we have in particular that $w_{k+1} \in C^{\infty}\left(B_{i}^{\prime}\right), w_{k+1}=w_{k}$ in $B_{i} \backslash B_{i}^{\prime \prime}$, and $\mathcal{H}^{n-1}\left(J_{w_{k}} \cap\right.$ $\left.\partial B_{i}^{\prime \prime}\right)=\mathcal{H}^{n-1}\left(J_{w_{k+1}} \cap \partial B_{i}^{\prime \prime}\right)=0$.

Property (2) of Lemma 3.1 provides a control on the (possible) additional jump of $w_{k+1}$ in each $B_{i}$ (note that this additional jump can only be in $B_{i}^{\prime \prime} \backslash B_{i}^{\prime}$ by Property (1)):

$$
\begin{aligned}
\mathcal{H}^{n-1}\left(\left(J_{w_{k+1}} \backslash J_{w_{k}}\right) \cap B_{i}\right) & =\mathcal{H}^{n-1}\left(\left(J_{w_{k+1}} \backslash J_{w_{k}}\right) \cap\left(B_{i}^{\prime \prime} \backslash B_{i}^{\prime}\right)\right) \\
& \leq c \eta_{k}^{\frac{1}{2 n}} \mathcal{H}^{n-1}\left(J_{w_{k}} \cap\left(B_{i} \backslash B_{i}^{\prime}\right)\right) .
\end{aligned}
$$

Here $c$ depends only on $n$ and $p$. We now estimate the jump of $w_{k+1}$ in each $B_{i}$. By property (1) of Lemma 3.1 and by (3.12)

$$
\begin{aligned}
\mathcal{H}^{n-1}\left(J_{w_{k+1}} \cap B_{i}\right) & =\mathcal{H}^{n-1}\left(J_{w_{k+1}} \cap\left(B_{i} \backslash B_{i}^{\prime}\right)\right) \\
& \leq \mathcal{H}^{n-1}\left(\left(J_{w_{k+1}} \backslash J_{w_{k}}\right) \cap\left(B_{i}^{\prime \prime} \backslash B_{i}^{\prime}\right)\right)+\mathcal{H}^{n-1}\left(J_{w_{k}} \cap\left(B_{i} \backslash B_{i}^{\prime}\right)\right) \\
& \leq\left(1+c \eta_{k}^{\frac{1}{2 n}}\right) \mathcal{H}^{n-1}\left(J_{w_{k}} \cap\left(B_{i} \backslash B_{i}^{\prime}\right)\right) .
\end{aligned}
$$

For the last term in (3.13) we have the bound

$$
\begin{aligned}
\mathcal{H}^{n-1}\left(J_{w_{k}} \cap\left(B_{i} \backslash B_{i}^{\prime}\right)\right) & =\mathcal{H}^{n-1}\left(J_{w_{k}} \cap B_{i}\right)-\mathcal{H}^{n-1}\left(J_{w_{k}} \cap B_{i}^{\prime}\right) \\
& \leq \eta_{k} r_{i}^{n-1}-\eta_{k}\left(\left(1-\eta_{k}^{\frac{1}{2 n}}\right) r_{i}\right)^{n-1} \\
& =\left(1-\left(1-\eta_{k}^{\frac{1}{2 n}}\right)^{n-1}\right) \mathcal{H}^{n-1}\left(J_{w_{k}} \cap B_{i}\right),
\end{aligned}
$$


where we have used properties (3.4) and (3.5) for the radii of the balls of the covering. Hence from (3.13) we have

$$
\mathcal{H}^{n-1}\left(J_{w_{k+1}} \cap B_{i}\right) \leq\left(1+c \eta_{k}^{\frac{1}{2 n}}\right)\left(1-\left(1-\eta_{k}^{\frac{1}{2 n}}\right)^{n-1}\right) \mathcal{H}^{n-1}\left(J_{w_{k}} \cap B_{i}\right)
$$

Possibly reducing $\bar{\delta}$, we may assume that

$$
\left(1+c \eta_{k}^{\frac{1}{2 n}}\right)\left(1-\left(1-\eta_{k}^{\frac{1}{2 n}}\right)^{n-1}\right) \leq(1+c \sqrt{\bar{\delta}})\left(1-(1-\sqrt{\bar{\delta}})^{n-1}\right) \leq \frac{1}{2},
$$

so that

$$
\mathcal{H}^{n-1}\left(J_{w_{k+1}} \cap B_{i}\right) \leq \frac{1}{2} \mathcal{H}^{n-1}\left(J_{w_{k}} \cap B_{i}\right)
$$

Note that (3.14) and (3.5) imply immediately that

$$
\mathcal{H}^{n-1}\left(J_{w_{k+1}}\right) \leq \mathcal{H}^{n-1}\left(J_{w_{k}}\right)
$$

In addition, by (3.14) and (3.5) one has

$$
\begin{aligned}
& \mathcal{H}^{n-1}\left(J_{w_{k+1}} \cap B_{\rho_{k}}\right)-\mathcal{H}^{n-1}\left(J_{w_{k}} \cap B_{\rho_{k}}\right) \\
& \quad \leq \sum_{i \geq 1}\left(\mathcal{H}^{n-1}\left(J_{w_{k+1}} \cap B_{i}\right)-\mathcal{H}^{n-1}\left(J_{w_{k}} \cap B_{i}\right)\right) \\
& \quad \leq-\frac{1}{2} \sum_{i \geq 1} \mathcal{H}^{n-1}\left(J_{w_{k}} \cap B_{i}\right) \leq-\frac{1}{2 \xi(n)} \mathcal{H}^{n-1}\left(J_{w_{k}} \cap B_{\left(1-s_{k}\right) \rho_{k}}\right),
\end{aligned}
$$

where the last inequality follows from (3.6). We deduce from (3.16) and (3.9) that

$$
\begin{aligned}
\mathcal{H}^{n-1}\left(J_{w_{k+1}} \cap B_{\rho_{k}}\right) \leq & \left(1-\frac{1}{2 \xi(n)}\right) \mathcal{H}^{n-1}\left(J_{w_{k}} \cap B_{\rho_{k}}\right) \\
& +\frac{1}{2 \xi(n)} \mathcal{H}^{n-1}\left(J_{w_{k}} \cap\left(B_{\rho_{k}} \backslash B_{\left(1-s_{k}\right) \rho_{k}}\right)\right) \\
\leq & \left(1-\frac{\theta}{2 \xi(n)}\right) \mathcal{H}^{n-1}\left(J_{w_{k}} \cap B_{\rho_{k}}\right) .
\end{aligned}
$$

Hence, using the value (3.7) of $\theta$, we obtain that

$$
\mathcal{H}^{n-1}\left(J_{w_{k+1}} \cap B_{\left(1-s_{k}\right) \rho_{k}}\right) \leq \theta \mathcal{H}^{n-1}\left(J_{w_{k}} \cap B_{\rho_{k}}\right) \leq \theta \eta_{k}\left(s_{k} \rho_{k}\right)^{n-1}
$$

where in the last inequality we used (3.3). In conclusion, whether (3.8) be satisfied or not, one has that, by (3.10) and (3.15),

$$
\mathcal{H}^{n-1}\left(J_{w_{k+1}}\right) \leq \mathcal{H}^{n-1}\left(J_{w_{k}}\right)
$$


and that, by (3.11) and (3.17),

$$
\mathcal{H}^{n-1}\left(J_{w_{k+1}} \cap B_{\left(1-s_{k}\right) \rho_{k}}\right) \leq \theta \mathcal{H}^{n-1}\left(J_{w_{k}} \cap B_{\rho_{k}}\right) \leq \theta \eta_{k}\left(s_{k} \rho_{k}\right)^{n-1} .
$$

We now define $\lambda:=\left(\theta /\left(1-s_{0}\right)^{n-1}\right)^{1 / n}$; recalling the definition (3.2) of $s_{0}$, one can ensure that $\lambda \leq \sqrt[2 n]{\theta}<1$ by choosing $\tau$ small enough, namely, by requiring that

$$
\tau \leq \eta_{0}\left(1-\theta^{\frac{1}{2(n-1)}}\right)^{n-1}=(\alpha \bar{\delta})^{n}\left(1-\theta^{\frac{1}{2(n-1)}}\right)^{n-1},
$$

which depends on $n, p, \alpha$. Then, letting $\rho_{k+1}:=\left(1-s_{k}\right) \rho_{k}, \eta_{k+1}:=\lambda \eta_{k}, s_{k+1}:=\lambda s_{k}$, we deduce from (3.19) that

$$
\mathcal{H}^{n-1}\left(J_{w_{k+1}} \cap B_{\rho_{k+1}}\right) \leq \eta_{k+1}\left(s_{k+1} \rho_{k+1}\right)^{n-1},
$$

which is (3.3) at step $k+1$.

Step 2: Convergence of $\left(w_{k}\right)_{k \geq 0}$. We now start the construction of the exceptional set $\omega$ given in the statement. To this aim, for every $k \geq 0$ we introduce the set $\omega_{k}$ in the following way. If (3.8) is satisfied we let $\omega_{k}:=\emptyset$, and if not, we let $\omega_{k}:=\bigcup_{i \geq 1} \bar{B}_{i}$ (note that $\omega_{k} \subseteq B_{\rho_{k}}$ ). In both cases $\left\{w_{k} \neq w_{k+1}\right\} \subset \omega_{k}$ and we can estimate the perimeter of $\omega_{k}$, thanks to (3.3) and (3.4), as

$$
\begin{aligned}
\mathcal{H}^{n-1}\left(\partial^{*} \omega_{k}\right) & \leq n \gamma_{n} \sum_{i \geq 1} r_{i}^{n-1}=\frac{n \gamma_{n}}{\eta_{k}} \sum_{i \geq 1} \mathcal{H}^{n-1}\left(J_{w_{k}} \cap B_{i}\right) \\
& \leq \frac{n \gamma_{n}}{\eta_{k}} \mathcal{H}^{n-1}\left(J_{w_{k}} \cap B_{\rho_{k}}\right) \leq n \gamma_{n}\left(s_{k} \rho_{k}\right)^{n-1}
\end{aligned}
$$

where $n \gamma_{n}=\mathcal{H}^{n-1}\left(\mathbb{S}^{n-1}\right)$ (see notation (k) in Sect. 2).

We now estimate the $L^{p}$-norm of $e\left(w_{k+1}\right)$ in terms of the norm of $e\left(w_{k}\right)$. Again, this bound is trivial if (3.8) is satisfied. If not, thanks to point (3) in Lemma 3.1, we have that in each $B_{i}$ of the construction

$$
\int_{B_{i}}\left|e\left(w_{k+1}\right)\right|^{p} d x \leq\left(1+c \eta_{k}^{\frac{s}{n}}\right) \int_{B_{i}}\left|e\left(w_{k}\right)\right|^{p} d x
$$

for each $i \geq 1$. As a consequence, by the definition of $w_{k+1}$, also

$$
\int_{B_{\rho}}\left|e\left(w_{k+1}\right)\right|^{p} d x \leq\left(1+c \eta_{k}^{\frac{s}{n}}\right) \int_{B_{\rho}}\left|e\left(w_{k}\right)\right|^{p} d x .
$$

Repeating the construction for all $k \geq 1$ we obtain sequences $\left(w_{k}\right)_{k \geq 0},\left(s_{k}\right)_{k \geq 0}$, $\left(\eta_{k}\right)_{k \geq 0},\left(\rho_{k}\right)_{k \geq 0}$ and $\left(\omega_{k}\right)_{k \geq 0}$ with:

$$
\eta_{k}=\lambda^{k}(\alpha \bar{\delta})^{n}, \quad s_{k}=\lambda^{k} s_{0}, \quad \rho_{k}=\rho \prod_{\ell=0}^{k-1}\left(1-\lambda^{\ell} s_{0}\right) .
$$


Since $\left(\rho_{k}\right)_{k}$ is decreasing, there exists $\rho^{\prime}:=\lim _{k \rightarrow \infty} \rho_{k}$. We claim that $\rho^{\prime}$ is bounded away from zero. Indeed, using that $\left(1-t s_{0}\right) \geq\left(1-s_{0}\right)^{t}$ for $t \in(0,1)$ and $s_{0}<1$

$$
\rho^{\prime}=\rho \prod_{\ell=0}^{\infty}\left(1-\lambda^{\ell} s_{0}\right) \geq \rho \prod_{\ell=0}^{\infty}\left(1-s_{0}\right)^{\lambda^{\ell}}=\rho\left(1-s_{0}\right)^{\frac{1}{1-\lambda}} \geq \rho\left(1-s_{0}\right)^{\frac{1}{1-\sqrt[2 n]{\theta}}}
$$

since $\lambda \leq \sqrt[2 n]{\theta}$

Now we set, for any $\ell \geq 0, \tilde{\omega}_{\ell}:=\bigcup_{k \geq \ell} \omega_{k}$. Then, thanks to (3.21) and to (3.24),

$$
\begin{aligned}
\mathcal{H}^{n-1}\left(\partial^{*} \tilde{\omega}_{\ell}\right) & \leq \sum_{k \geq \ell} \mathcal{H}^{n-1}\left(\partial^{*} \omega_{k}\right) \leq n \gamma_{n} \sum_{k \geq \ell}\left(s_{k} \rho_{k}\right)^{n-1} \\
& \leq n \gamma_{n}\left(s_{\ell} \rho_{\ell}\right)^{n-1} \sum_{k \geq \ell}\left(\lambda^{k-\ell}\right)^{n-1} \leq n \gamma_{n}\left(s_{\ell} \rho_{\ell}\right)^{n-1} \frac{1}{1-\lambda^{n-1}},
\end{aligned}
$$

where we have used the fact that $\rho_{k} \leq \rho_{\ell}$ for $k \geq \ell$. Then, since $\rho_{\ell} \rightarrow \rho^{\prime}$ and $s_{\ell} \rightarrow 0$ as $\ell \rightarrow \infty$, it follows that $\mathcal{H}^{n-1}\left(\partial^{*} \tilde{\omega}_{\ell}\right) \rightarrow 0$ as $\ell \rightarrow \infty$. Hence by the isoperimetric inequality we also have that $\mathcal{L}^{n}\left(\tilde{\omega}_{\ell}\right) \rightarrow 0$ as $\ell \rightarrow \infty$. Since, for $k \geq \ell, w_{k}=w_{\ell}$ outside $\tilde{\omega}_{\ell}$, we conclude that, as $k \rightarrow \infty, w_{k}$ converges $\mathcal{L}^{n}$-a.e. in $B_{\rho}$ to some function $w$. We also note that, for every $k \geq 0$, by (3.23) and (3.24),

$$
\begin{aligned}
\int_{B_{\rho}}\left|e\left(w_{k}\right)\right|^{p} d x & \leq \prod_{i=0}^{k-1}\left(1+c \eta_{i}^{\frac{s}{n}}\right) \int_{B_{\rho}}|e(u)|^{p} d x \\
& \leq \prod_{i=0}^{\infty}\left(1+c(\alpha \bar{\delta})^{s} \lambda^{\frac{i s}{n}}\right) \int_{B_{\rho}}|e(u)|^{p} d x .
\end{aligned}
$$

Using $1+t \leq e^{t}$, we estimate

$$
\prod_{i=0}^{\infty}\left(1+c(\alpha \bar{\delta})^{s} \lambda^{\frac{i s}{n}}\right) \leq \exp \left(c(\alpha \bar{\delta})^{s} \sum_{i \geq 0}\left(\lambda^{\frac{s}{n}}\right)^{i}\right)=\exp \left(c(\alpha \bar{\delta})^{s} \frac{1}{1-\lambda^{\frac{s}{n}}}\right)
$$

so that

$$
\int_{B_{\rho}}\left|e\left(w_{k}\right)\right|^{p} d x \leq \exp \left(c(\alpha \bar{\delta})^{s} \frac{1}{1-\lambda^{\frac{s}{n}}}\right) \int_{B_{\rho}}|e(u)|^{p} d x
$$

Moreover, thanks to (3.18), for every $k \geq 0$ we have that $\mathcal{H}^{n-1}\left(J_{w_{k}}\right) \leq \mathcal{H}^{n-1}\left(J_{u}\right)$. Then, thanks to [22, Theorem 11.3] (see also [12]) it follows that $w \in G S B D^{p}\left(B_{\rho}\right)$,

$$
\mathcal{H}^{n-1}\left(J_{w}\right) \leq \liminf _{k \rightarrow \infty} \mathcal{H}^{n-1}\left(J_{w_{k}}\right) \leq \mathcal{H}^{n-1}\left(J_{u}\right)
$$


and

$$
\int_{B_{\rho}}|e(w)|^{p} d x \leq \liminf _{k \rightarrow \infty} \int_{B_{\rho}}\left|e\left(w_{k}\right)\right|^{p} d x \leq \exp \left(c(\alpha \bar{\delta})^{s} \frac{1}{1-\lambda^{\frac{s}{n}}}\right) \int_{B_{\rho}}|e(u)|^{p} d x .
$$

Passing to the limit in (3.3) we have that $\mathcal{H}^{n-1}\left(J_{w} \cap B_{\rho^{\prime}}\right)=0$, from which it follows that $w \in W^{1, p}\left(B_{\rho^{\prime}} ; \mathbb{R}^{n}\right)$, thanks to Korn's inequality. Note that by (3.24) $\rho^{\prime} \leq \rho$, and $\rho^{\prime} \rightarrow \rho$ as $\mathcal{H}^{n-1}\left(J_{u}\right) \rightarrow 0$, thanks to (3.25) (and by the definition of $\left.s_{0}\right)$. Clearly, using (3.25) and choosing $\tau$ small enough we can ensure

$$
\rho \geq \rho\left(1-s_{0}\right)^{\frac{1}{1-\sqrt[2 n]{\theta}}} \geq \rho(1-\sigma)
$$

This holds, for instance, for

$$
\tau:=\eta_{0}(1-\sqrt[2 n]{\theta})^{n-1} \sigma^{n-1}
$$

which also satisfies (3.20). Here we used that $(1-\sqrt[2 n]{\theta}) \sigma \leq 1-(1-\sigma)^{1-\sqrt[2 n]{\theta}}$. Note that $\tau=\tau(n, p, \alpha, \sigma)$. With this choice we obtain that $w \in W^{1, p}\left(B_{\rho(1-\sigma)} ; \mathbb{R}^{n}\right)$.

Setting $\omega:=\tilde{\omega}_{0}$, by construction we have that $w=u$ in $B_{\rho} \backslash \omega$. In addition, from (3.26) we have that $\mathcal{H}^{n-1}\left(\partial^{*} \omega\right) \leq n \gamma_{n}\left(s_{0} \rho\right)^{n-1} /\left(1-\lambda^{n-1}\right)$. By our choice (3.2) of $s_{0}$, this implies

$$
\mathcal{H}^{n-1}\left(\partial^{*} \omega\right) \leq \frac{n \gamma_{n}}{(\alpha \bar{\delta})^{n}\left(1-\lambda^{n-1}\right)} \mathcal{H}^{n-1}\left(J_{u}\right)
$$

Using the fact that $\lambda \leq \sqrt[2 n]{\theta}$ we finally obtain the estimates

$$
\begin{gathered}
\int_{B_{\rho}}|e(w)|^{p} d x \leq \exp \left(\frac{c(\alpha \bar{\delta})^{s}}{1-\theta^{\frac{s}{n^{2}}}}\right) \int_{B_{\rho}}|e(u)|^{p} d x, \\
\mathcal{H}^{n-1}\left(\partial^{*} \omega\right) \leq \frac{n \gamma_{n}}{(\alpha \bar{\delta})^{n}\left(1-\theta^{\frac{n-1}{2 n}}\right)} \mathcal{H}^{n-1}\left(J_{u}\right) .
\end{gathered}
$$

Now we choose $\alpha=\alpha(n, p, \varepsilon) \in(0,1)$ such that

$$
\exp \left(\frac{c(\alpha \bar{\delta})^{s}}{1-\theta^{\frac{s}{2 n^{2}}}}\right) \leq(1+\varepsilon)
$$

Note that now $\tau=\tau(n, p, \varepsilon, \sigma)$. Correspondingly, we define

$$
C=C(n, p, \varepsilon):=\frac{n \gamma_{n}}{(\alpha \bar{\delta})^{n}\left(1-\theta^{\frac{n-1}{2 n}}\right)}
$$

as the constant in the statement of the theorem, and this concludes the proof. 
Remark 3.5 From (3.22), it is easy to show that in fact one can refine (3.1) to

$$
\int_{\omega}|e(w)|^{p} d x \leq(1+\varepsilon) \int_{\omega}|e(u)|^{p} d x .
$$

In addition, one sees that $C \sim \varepsilon^{-n / s}$, where $s$ is the exponent in Property (3) of Lemma 3.1.

Remark 3.6 It is easy to show (by modifying the proof or, in fact, using the theorem itself) a variant of Theorem 3.2 where $B_{\rho}$ is replaced with a cube $(-\rho, \rho)^{n}$.

We can easily deduce that [16, Corollary 3.3] also holds in higher dimension. We repeat the statement here for the reader's convenience.

Corollary 3.7 Under the same assumptions and notation of Theorem 3.2, there exists an infinitesimal rigid motion $a \in \mathcal{R}$ such that

$$
\int_{B_{(1-\sigma) \rho} \backslash \omega}|\nabla u-\nabla a|^{p} d x \leq c(n, p) \int_{B_{\rho}}|e(u)|^{p} d x,
$$

and

$$
\int_{B_{(1-\sigma) \rho} \backslash \omega}|u-a|^{p} d x \leq c(n, p) \rho^{p} \int_{B_{\rho}}|e(u)|^{p} d x .
$$

\section{Regularity and rigidity in a general domain}

The main result of this section is the following regularity result.

Theorem 4.1 Let $n \in \mathbb{N}$ with $n \geq 2, p \in(1, \infty)$, and let $\Omega \subset \mathbb{R}^{n}$ be a bounded and open Lipschitz set. There exists $c=c(n, p, \Omega)>0$ such that, for any $u \in$ $G S B D^{p}(\Omega)$, there is a set of finite perimeter $\omega \subset \Omega$ with $\mathcal{H}^{n-1}\left(\partial^{*} \omega\right) \leq c \mathcal{H}^{n-1}\left(J_{u}\right)$ and $v \in W^{1, p}\left(\Omega ; \mathbb{R}^{n}\right)$ such that $u=v$ in $\Omega \backslash \omega$ and $\int_{\Omega}|e(v)|^{p} d x \leq c \int_{\Omega}|e(u)|^{p} d x$. If in addition $u$ is bounded, then $\|v\|_{L^{\infty}(\Omega)} \leq\|u\|_{L^{\infty}(\Omega)}$. The constant $c$ is invariant under uniform scalings of the domain.

Remark 4.2 Note that the conclusion of Theorem 4.1 is non-trivial only when the measure of the jump set $J_{u}$ is small, since otherwise one can simply take $\omega:=\Omega$ and $v:=0$ (see the proof of Theorem 4.1 for more details).

Remark 4.3 A careful inspection of the proof of Theorem 4.1 shows that the constant $c=c(n, p, \Omega)$ depends on $\Omega$ via the triple $(N, r, L)$ defined as follows:

(i) for every $x \in \partial \Omega$ there exists $\mathrm{e}(x) \in \mathbb{S}^{n-1}$ such that $\partial \Omega \cap C(x, \mathrm{e}(x), 4 \operatorname{Lr}, 2 r)$ is the graph of an $L$-Lipschitz function defined on the $(n-1)$-dimensional ball $\left\{y \in \Pi_{x}^{\mathrm{e}(x)}:|(y-x)-((y-x) \cdot \mathrm{e}(x)) \mathrm{e}(x)|<2 r\right\}$

(ii) there exist $N$ points $x_{1}, \ldots, x_{N} \in \partial \Omega$ such that $\partial \Omega \subset \bigcup_{i=1}^{N} B_{r}\left(x_{i}\right)$ and $B_{r / 5}\left(x_{1}\right), \ldots, B_{r / 5}\left(x_{N}\right)$ are mutually disjoint. 
Note that property (i) follows from the fact that $\Omega$ is a Lipschitz domain, while the existence of $N$ satisfying property (ii) is shown in the proof of Theorem 4.1. Moreover, $N$ satisfies the estimate

$$
N \gamma_{n-1}(r / 5)^{n-1} \leq \mathcal{H}^{n-1}(\partial \Omega) \leq N \gamma_{n-1} r^{n-1} \sqrt{1+L^{2}}
$$

For the proof of Theorem 4.1 we will use the following lemma.

Lemma 4.4 Let $n \in \mathbb{N}$ with $n \geq 2, p \in(1, \infty)$, and let $D \subset \mathbb{R}^{n}$ be a bounded, open and connected Lipschitz set. Let $\alpha \in(0,1)$. There exist $c>0$ depending only on $D, \alpha$ and $p$, such that for any $w \in W^{1, p}\left(D ; \mathbb{R}^{n}\right)$ and any Lebesgue measurable set $E \subset D$ with $\mathcal{L}^{n}(E) \geq \alpha \mathcal{L}^{n}(D)$, one has

$$
\int_{D}\left|w-a_{E}\right|^{p} d x \leq c \int_{D}|e(w)|^{p} d x
$$

where

$$
a_{E}:=\arg \min _{a \in \mathcal{R}} \int_{E}|w-a|^{p} d x .
$$

Proof Such a lemma is standard and easily proved by contradiction. Suppose that for every $k \in \mathbb{N}$ there exist a function $w_{k} \in W^{1, p}\left(D ; \mathbb{R}^{n}\right)$ and a Lebesgue measurable set $E_{k} \subset D$ with $\mathcal{L}^{n}\left(E_{k}\right) \geq \alpha \mathcal{L}^{n}(D)$ such that

$$
\int_{D}\left|w_{k}-a_{E_{k}}\right|^{p} d x>k \int_{D}\left|e\left(w_{k}\right)\right|^{p} d x,
$$

where $a_{E_{k}}$ is as in (4.1). Setting

$$
u_{k}:=\frac{w_{k}-a_{E_{k}}}{\left\|w_{k}-a_{E_{k}}\right\|_{L^{p}\left(D ; \mathbb{R}^{n}\right)}},
$$

we have that, by the definition of $a_{E_{k}}, u_{k}$ satisfies

$$
\int_{E_{k}}\left|u_{k}\right|^{p} d x \leq \int_{E_{k}}\left|u_{k}-a\right|^{p} d x \quad \forall a \in \mathcal{R} .
$$

Moreover, by (4.2),

$$
\left\|u_{k}\right\|_{L^{p}\left(D ; \mathbb{R}^{n}\right)}=1, \quad\left\|e\left(u_{k}\right)\right\|_{L^{p}\left(D ; \mathbb{R}_{\mathrm{sym}}^{n \times n}\right)}^{p}<\frac{1}{k},
$$

so that by the classical Korn inequality

$$
\left\|u_{k}\right\|_{W^{1, p}\left(D ; \mathbb{R}^{n}\right)} \leq C\left(\left\|u_{k}\right\|_{L^{p}\left(D ; \mathbb{R}^{n}\right)}+\left\|e\left(u_{k}\right)\right\|_{L^{p}\left(D ; \mathbb{R}_{\mathrm{sym}}^{n \times n}\right)}\right) \leq 2 C,
$$


for some constant $C=C(n, p, D)>0$. Hence, there exists $u \in W^{1, p}\left(D ; \mathbb{R}^{n}\right)$ such that, up to subsequences, $u_{k} \rightarrow u$ weakly in $W^{1, p}\left(D ; \mathbb{R}^{n}\right)$ as $k \rightarrow+\infty$ (and strongly in $L^{p}\left(D ; \mathbb{R}^{n}\right)$ ). Note that, up to subsequences, the characteristic functions $\chi_{E_{k}}$ of $E_{k}$ converge weakly* in $L^{\infty}(D)$ to some function $\phi \in L^{\infty}(D)$ with $0 \leq \phi \leq 1$ and such that

$$
\alpha \mathcal{L}^{n}(D) \leq \int_{D} \phi d x
$$

Therefore, passing to the limit in (4.3) and (4.4), we have that $e(u)=0$ in $D$ and

$$
\int_{D}|u|^{p} \phi d x \leq \int_{D}|u-a|^{p} \phi d x \quad \forall a \in \mathcal{R}, \quad\|u\|_{L^{p}\left(D ; \mathbb{R}^{n}\right)}=1 .
$$

Since $e(u)=0$ in $D$, by the classical Poincaré-Korn inequality (see, e.g., (1.6)) we deduce that there exists $\bar{a} \in \mathcal{R}$ such that $u=\bar{a}$ in $D$. Choosing $a=\bar{a}$ in (4.6) we then have

$$
\int_{D}|\bar{a}|^{p} \phi d x=0 .
$$

Since $\bar{a} \in \mathcal{R}$ and taking into account (4.5) it follows that $\bar{a}=0$ and hence $u=0$. This is however incompatible with $\|u\|_{L^{p}\left(D ; \mathbb{R}^{n}\right)}=1$.

Proof [Proof of Theorem 4.1] It is enough to prove the result in the case $\mathcal{H}^{n-1}\left(J_{u}\right) \leq \bar{c}$, for some constant $\bar{c}>0$ to be determined later on (see (4.9)). Indeed, if $\mathcal{H}^{n-1}\left(J_{u}\right)>\bar{c}$, we can simply set $\omega:=\Omega$ and $v:=0$, which clearly satisfy the required bounds, since $\mathcal{H}^{n-1}\left(\partial^{*} \omega\right) \leq\left(\mathcal{H}^{n-1}\left(\partial^{*} \Omega\right) / \bar{c}\right) \mathcal{H}^{n-1}\left(J_{u}\right)$.

We divide the proof into several steps.

Step 1: We introduce a finite open cover $\left\{C_{0}, C_{1}, \ldots, C_{N}\right\}$ of $\bar{\Omega}$. Since $\Omega$ is Lipschitz and bounded (see [1, Sect. 4.9]), there exist $r>0$ such that for every $x \in \partial \Omega$, there exists $\mathrm{e}(x) \in \mathbb{S}^{n-1}$ such that $\partial \Omega \cap C(x, \mathrm{e}(x), 4 L r, 2 r)$ (see (d) in the Notation Section) is the graph of an $L$-Lipschitz function defined on the $(n-1)$-dimensional ball $\left\{y \in \Pi_{x}^{\mathrm{e}(x)}:|(y-x)-((y-x) \cdot \mathrm{e}(x)) \mathrm{e}(x)|<2 r\right\}$. Setting $\tilde{L}:=\max \{1, L\}$, by possibly reducing $r$ we still have that $\partial \Omega \cap C(x, \mathrm{e}(x), 4 \tilde{L} r, 2 r)$ is the graph of an $\tilde{L}$-Lipschitz function for every $x \in \partial \Omega$. Consider now the family of open balls $\left\{B_{r / 5}(x)\right\}_{x \in \partial \Omega}$. By Vitali's Covering Theorem [24, Section 1.5.1], there exist $N \in$ $\mathbb{N}$ and $\left\{x_{1}, \ldots, x_{N}\right\} \subset \partial \Omega$ such that the family $\left\{B_{r / 5}\left(x_{i}\right)\right\}_{i=1, \ldots, N}$ is composed of mutually disjoint balls and

$$
\partial \Omega \subset \bigcup_{x \in \partial \Omega} B_{r / 5}(x) \subset \bigcup_{i=1}^{N} B_{r}\left(x_{i}\right) .
$$

In the following, we will use the shorthand $C_{i}:=C\left(x_{i}, \mathrm{e}\left(x_{i}\right), 4 \tilde{L} r, 2 r\right)$ for every $i=1, \ldots, N$. Note that $B_{r}\left(x_{i}\right) \subset B_{2 r}\left(x_{i}\right) \subset C_{i}$ and that $\operatorname{dist}\left(\partial C_{i}, B_{r}\left(x_{i}\right)\right)=r$ for every $i=1, \ldots, N$. Moreover, from (4.7) it follows that 


$$
\left\{x \in \Omega: \operatorname{dist}(x, \partial \Omega) \leq \frac{r}{6}\right\} \subset\left\{x \in \Omega: \operatorname{dist}(x, \partial \Omega)<\frac{r}{5}\right\} \subset \bigcup_{i=1}^{N} B_{r}\left(x_{i}\right)
$$

Setting

$$
\widetilde{C}_{0}:=\left\{x \in \Omega: \operatorname{dist}(x, \partial \Omega)>\frac{r}{6}\right\}, \quad C_{0}:=\left\{x \in \Omega: \operatorname{dist}(x, \partial \Omega)>\frac{r}{8}\right\},
$$

thanks to (4.8) it follows that $\bar{\Omega} \subset \widetilde{C}_{0} \cup\left(\bigcup_{i=1}^{N} B_{r}\left(x_{i}\right)\right) \subset \bigcup_{i=0}^{N} C_{i}$.

Step 2: We show that, at any given point of $\Omega$, the maximal number of overlapping sets in the covering $\left\{C_{0}, C_{1}, \ldots, C_{N}\right\}$ only depends on $L$ and $n$. To this aim, it will be enough to prove the statement for the sets $C_{1}, \ldots, C_{N}$. Let $z \in \Omega$, and let

$$
A(z):=\left\{x \in\left\{x_{1}, \ldots, x_{N}\right\}: z \in C(x, \mathrm{e}(x), 4 \tilde{L} r, 2 r)\right\} .
$$

Our goal is to show that the cardinality of $A(z)$ is bounded by a number that only depends on $n$ and $L$. Note that, if $z$ is 'far' from $\partial \Omega$, it can be $A(z)=\emptyset$, but in this case there is nothing to prove. Since the diameter of each cylinder is given by $4 r \sqrt{1+4 \tilde{L}^{2}}$, we have

$$
|z-x|<4 r \sqrt{1+4 \tilde{L}^{2}} \text { for every } x \in A(z)
$$

Therefore,

$$
A(z) \subset B_{4 r} \sqrt{1+4 \tilde{L}^{2}}(z)
$$

Recalling that the family $\left\{B_{r / 5}\left(x_{i}\right)\right\}_{i=1, \ldots, N}$ is composed of mutually disjoint balls, for every $i \in\{1, \ldots, N\}$ with $i \neq j$ we have $\left|x_{i}-x_{j}\right|>2 r / 5$. Then, the cardinality of $A(z)$ is bounded by the maximum number of disjoint balls of radius $r / 5$ which can intersect a ball of radius $4 r \sqrt{1+4 \tilde{L}^{2}}$, that we denote with $\kappa$. By scaling, one can check that $\kappa$ does not depend on $r$, but only on $\tilde{L}$ (i.e. on $L$ ) and on the dimension $n$. Step 3: We show that it is enough to prove the theorem in the set $C_{i} \cap \Omega$, for every $i \in\{0,1 \ldots, N\}$. We introduce a partition of unity of $\Omega$ subordinate to the open covering $\left\{\widetilde{C}_{0}, B_{r}\left(x_{1}\right), \ldots, B_{r}\left(x_{N}\right)\right\}$, namely maps $\phi_{0} \in C_{c}^{\infty}\left(\widetilde{C}_{0},[0,1]\right)$, and $\phi_{i} \in$ $C_{c}^{\infty}\left(B_{r}\left(x_{i}\right),[0,1]\right)$ for $i=1, \ldots, N$, with $\sum_{i=0}^{N} \phi_{i}=1$ in $\Omega$ (see, e.g. [30, Definition A.13]). Note that this is also a partition of unity of $\Omega$ subordinate to the open covering $\left\{C_{i}\right\}$, for $i=0, \ldots, N$. Moreover, by construction $\operatorname{dist}\left(\operatorname{supp} \phi_{0}, \partial C_{0}\right)>r / 24$, while $\operatorname{dist}\left(\operatorname{supp} \phi_{i}, \partial C_{i}\right) \geq r$ for $i=1, \ldots, N$. Assuming that for every $i$ we can find a function $v_{i}$ and a set $\omega_{i}$ satisfying the thesis of the theorem in $C_{i} \cap \Omega$, then $v=$ $\sum_{i=0}^{N}\left(\phi_{i \mid C_{i} \cap \Omega}\right) v_{i}$ and $\omega=\bigcup_{i=0}^{N} \omega_{i}$ satisfy the claim in $\Omega$.

Step 4: We fix $i \in\{0,1, \ldots, N\}$ and prove the theorem in $C_{i} \cap \Omega$. Let $i \in\{0,1, \ldots, N\}$. Our construction will be simpler in the case $i=0$ and, when necessary, we will explicitly point this out in the proof. If $i \neq 0$, without loss of generality we can assume that $C_{i}=C\left(0, e_{n}, 4 \tilde{L} r, 2 r\right)$, with $e_{n}$ being the $n$-th coordinate unit vector, 
and that $\Omega \cap C_{i}=\left\{x=\left(x^{\prime}, x_{n}\right) \in C_{i}: x_{n}<g\left(x^{\prime}\right)\right\}$ for a given $\tilde{L}$-Lipschitz function $g$ defined on the $\left((n-1)\right.$-dimensional) ball centred at 0 and of radius $r$ in $\Pi_{0}^{e_{n}}$, with $g(0)=0$.

We now build $v_{i}$ and $\omega_{i}$ for the set $C_{i} \cap \Omega$. Let $\delta>0$, and let $\mathbf{C}_{i}$ denote the union of all the $n$-dimensional cubes $q \in\left\{z+(0, \delta]^{n}: z \in \delta \mathbb{Z}^{n}\right\}$ with $q \subset C_{i}$. Since $\operatorname{dist}\left(\operatorname{supp} \phi_{i}, \partial C_{i}\right)>r / 24$, we can assume that $\delta$ is small enough so that supp $\phi_{i} \subset \mathbf{C}_{i}$. Note that the choice of $\delta / r$ depends only on $n$.

Then we build recursively the set $\mathcal{Q}$ of dyadic cubes of edge size $\delta 2^{-k}, k \geq 0$, which refine towards the boundary $\partial \Omega$, as follows. As a first step, we denote with $\mathcal{Q}_{0}$ the set of cubes $q \in\left\{z+(0, \delta]^{n}: z \in \delta \mathbb{Z}^{n}\right\}, q \subset \mathbf{C}_{i} \cap \Omega$, such that $\operatorname{dist}(q, \partial \Omega)>\delta$. Then, for $k \geq 1$, having built $\mathcal{Q}_{\ell}$ for $\ell<k$, we define $\mathcal{Q}_{k}$ as the set of all the smaller cubes $q \in\left\{z+\left(0, \delta 2^{-k}\right]^{n}: z \in \delta 2^{-k} \mathbb{Z}^{n}\right\}, q \subset \mathbf{C}_{i} \cap \Omega$, such that $\operatorname{dist}(q, \partial \Omega)>\delta 2^{-k}$, and $q$ does not intersect cubes of $\bigcup_{\ell<k} \bigcup_{\hat{q} \in \mathcal{Q}_{\ell}} \hat{q}$. Note that, if $i=0$, we can assume that all the cubes in $\mathbf{C}_{0}$ belong to the family $\mathcal{Q}_{0}$ (by e.g. choosing $\delta<r / 8$ ).

Finally, we let $\mathcal{Q}:=\bigcup_{k=0}^{\infty} \mathcal{Q}_{k}$; note that $\bigcup_{q \in \mathcal{Q}} q=\mathbf{C}_{i} \cap \Omega \subset C_{i} \cap \Omega$ covers entirely supp $\phi_{i} \cap \Omega$. Now, for each $q \in \mathcal{Q}$, let $q^{\prime}$ and $q^{\prime \prime}$ denote cubes concentric with $q$, and with edge size $10 \%$ and $20 \%$ longer, respectively. Then the cubes $q^{\prime \prime}$ (as well as $q^{\prime}$ ), for $q \in \mathcal{Q}$, form a sort of Whitney covering of $C_{i} \cap \Omega$, at least covering supp $\phi_{i} \cap \Omega$. Moreover, since for every $k \geq 0$ any $q \in \mathcal{Q}_{k}$ satisfies $\operatorname{dist}(q, \partial \Omega)>\delta 2^{-k}$, clearly also $q^{\prime}, q^{\prime \prime} \subset \Omega$. Note that, for fixed $k \geq 0$, an enlarged cube $q^{\prime \prime}$ of some cube $q \in \mathcal{Q}_{k}$ can only intersect cubes belonging to $\mathcal{Q}_{k}, \mathcal{Q}_{k+1}$ and, if $k \geq 1, \mathcal{Q}_{k-1}$.

Next, we choose the constant $\bar{c}=\bar{c}(\Omega)$ introduced at the start of the proof to be

$$
\bar{c}:=\tau(\delta / 2)^{n-1},
$$

where $\tau$ is given by Theorem 3.2 (or, more precisely, by the version of Theorem 3.2 for a cube, following Remark 3.6), corresponding to $\sigma=1 / 12$ and $\varepsilon=1$. Hence, by the initial assumption $\mathcal{H}^{n-1}\left(J_{u}\right) \leq \bar{c}$ we have

$$
\mathcal{H}^{n-1}\left(J_{u}\right) \leq \tau(\delta / 2)^{n-1} .
$$

Then, by applying Theorem 3.2 (with $\varepsilon=1$ ) to $u \in G S B D^{p}\left(q^{\prime \prime}\right)$, for each $q \in \mathcal{Q}_{0}$, we find a function $w_{q} \in \operatorname{GSB} D^{p}\left(q^{\prime \prime}\right)$ and a set of finite perimeter $\omega_{q} \subset q^{\prime \prime}$ such that $w_{q}=$ $u$ in $q^{\prime \prime} \backslash \omega_{q}, \int_{q^{\prime \prime}}\left|e\left(w_{q}\right)\right|^{p} d x \leq C \int_{q^{\prime \prime}}|e(u)|^{p} d x, \mathcal{H}^{n-1}\left(\partial^{*} \omega_{q}\right) \leq C \mathcal{H}^{n-1}\left(J_{u} \cap q^{\prime \prime}\right)$ and $w_{q} \in W^{1, p}\left(q^{\prime} ; \mathbb{R}^{n}\right)$, where $C=C(n, p)$.

For smaller cubes $q \in \mathcal{Q}_{k}, k \geq 1$, we proceed as follows: if $\mathcal{H}^{n-1}\left(J_{u} \cap q^{\prime \prime}\right) \leq$ $\tau\left(\delta / 2^{k+1}\right)^{n-1}$, we say that $q$ is "good", we apply Theorem 3.2 to the restriction of $u$ to $q^{\prime \prime}$, and find $w_{q}$ and $\omega_{q}$ as in the case $k=0$ (note that all the cubes in $\mathcal{Q}_{0}$ are "good" and that, in particular, $\mathbf{C}_{0}$ is made of "good" cubes). In conclusion, for $q$ "good", we find a function $w_{q} \in G S B D^{p}\left(q^{\prime \prime}\right)$ and a set of finite perimeter $\omega_{q} \subset q^{\prime \prime}$ such that $w_{q}=u$ in $q^{\prime \prime} \backslash \omega_{q}$ and

$$
\begin{aligned}
& w_{q} \in W^{1, p}\left(q^{\prime} ; \mathbb{R}^{n}\right), \\
& \int_{q^{\prime \prime}}\left|e\left(w_{q}\right)\right|^{p} d x \leq C \int_{q^{\prime \prime}}|e(u)|^{p} d x,
\end{aligned}
$$




$$
\mathcal{H}^{n-1}\left(\partial^{*} \omega_{q}\right) \leq C \mathcal{H}^{n-1}\left(J_{u} \cap q^{\prime \prime}\right),
$$

where $C=C(n, p)$. If instead $\mathcal{H}^{n-1}\left(J_{u} \cap q^{\prime \prime}\right)>\tau\left(\delta / 2^{k+1}\right)^{n-1}$, we say that $q$ is "bad" and we define

$$
\tilde{\omega}_{q}:=\Omega \cap\left(q+\left\{x=\left(x^{\prime}, x_{n}\right): x_{n}>2 \tilde{L}\left|x^{\prime}\right|\right\}\right)
$$

namely we connect $q$ with $\partial \Omega$ via a sort of truncated cone with an opening controlled by the Lipschitz constant $L$ of $\Omega$. Scaling arguments (and the fact that $\left.\operatorname{dist}(q, \partial \Omega) \leq \delta 2^{-k+1}\right)$ show that $\mathcal{H}^{n-1}\left(\partial^{*} \tilde{\omega}_{q}\right) \leq c\left(\delta 2^{-k}\right)^{n-1}$ where the constant $c=c(n, L)$ depends only on $L$ and the dimension. It follows that in this case, namely for $q$ "bad",

$$
\mathcal{H}^{n-1}\left(\partial^{*} \tilde{\omega}_{q}\right) \leq c(n, L) \frac{2^{n-1}}{\tau} \mathcal{H}^{n-1}\left(J_{u} \cap q^{\prime \prime}\right) .
$$

We let $\tilde{\omega}:=\bigcup_{q \in \mathcal{Q}_{b}} \tilde{\omega}_{q}, G:=\left(\bigcup_{q \in \mathcal{Q}} q\right) \backslash \tilde{\omega}$, and $\hat{\omega}:=\bigcup_{q \in \mathcal{Q}_{g}}\left(\omega_{q} \cap q^{\prime}\right)$, where we denoted with $\mathcal{Q}_{b}, \mathcal{Q}_{g} \subset \mathcal{Q}$ the "bad" and "good" cubes in $\mathcal{Q}$, respectively. By construction, there exists a $(2 \tilde{L})$-Lipschitz function $f$ such that $G$ is the subgraph of $f$, with $g-2 \delta \leq f \leq g$. Moreover, for some constant $c$ (depending on $L, n$ and $\tau$ ), and using that the cubes $q^{\prime \prime}$ have finite overlap, one has, by (4.12) and (4.13),

$$
\mathcal{H}^{n-1}\left(\partial^{*} \omega_{i}\right) \leq \mathcal{H}^{n-1}\left(\partial^{*} \tilde{\omega}\right)+\mathcal{H}^{n-1}\left(\partial^{*} \hat{\omega}\right) \leq c \mathcal{H}^{n-1}\left(J_{u} \cap\left(C_{i} \cap \Omega\right)\right),
$$

where we set $\omega_{i}:=\tilde{\omega} \cup \hat{\omega}$.

We now construct a regularised function $v_{i}$ as a convex combination of the functions $w_{q}$ relative to "good" cubes $q \in \mathcal{Q}_{g}$ only. More precisely, let $\psi \in$ $C_{c}^{\infty}\left((0,1.1)^{n} ;[0,1]\right)$ be a smooth cut-off function with $\psi=1$ on $[0,1]^{n}$. For any $k \geq 0$ and any $q \in \mathcal{Q}_{g} \cap \mathcal{Q}_{k}$ with centre $c_{q}$, we define the translated and rescaled version of $\psi_{q}, \psi_{q}(x):=\psi\left(\left(x-c_{q}\right) /\left(\delta 2^{-k}\right)\right) \in C_{c}^{\infty}\left(q^{\prime} ;[0,1]\right)$, so that $\psi_{q}=1$ on $q$. Finally, we define the 'normalised' cut-off function $\varphi_{q}(x):=\psi_{q}(x) /\left(\sum_{\hat{q} \in \mathcal{Q}_{g}} \psi_{\hat{q}}(x)\right)$ for $x \in \cup_{q \in \mathcal{Q}_{g}} q$.

We then let, for $x \in \cup_{q \in \mathcal{Q}_{g}} q, \tilde{v}_{i}(x):=\sum_{q \in \mathcal{Q}_{g}} w_{q}(x) \varphi_{q}(x)$. First of all, we extend $\tilde{v}_{i \mid G}$ from $G$ to $\mathbf{C}_{i} \cap \Omega$. This can be done, for instance, by following the procedure in [31, Lemma 4], since $G$ is a special Lipschitz set (according to [31, property (49)]) and $\tilde{v}_{i \mid G} \in W^{1, p}\left(G ; \mathbb{R}^{n}\right)$, as each $w_{q}$ belongs to $W^{1, p}\left(q^{\prime} ; \mathbb{R}^{n}\right)$ for $q \in \mathcal{Q}_{g}$, by (4.10). We denote this extension by $v_{i}$. Then $v_{i} \in W^{1, p}\left(\mathbf{C}_{i} \cap \Omega ; \mathbb{R}^{n}\right), v_{i}=\tilde{v}_{i}$ in $G$, and by $[31$, property $(50)]$ we have that

$$
\int_{\mathbf{C}_{i} \cap \Omega}\left|e\left(v_{i}\right)\right|^{p} d x \leq c \int_{G}\left|e\left(\tilde{v}_{i}\right)\right|^{p} d x \leq c \int_{\cup_{q \in \mathcal{Q}_{g}} q}\left|e\left(\tilde{v}_{i}\right)\right|^{p} d x,
$$

where the constant $c$ depends only on the dimension $n$, on $p$, and on the Lipschitz constant of $G$ (namely of $f$ ), which is $2 \tilde{L}$, hence $c=c(n, p, L)$.

Moreover, $v_{i}=u$ in $\left(\mathbf{C}_{i} \cap \Omega\right) \backslash \omega_{i}$. Indeed, $\tilde{v}_{i \mid G}=u$ in $G \backslash \hat{\omega}$ by construction, $v_{i}=\tilde{v}_{i}$ in $G$, and $G=\left(\mathbf{C}_{i} \cap \Omega\right) \backslash \tilde{\omega}$. 
To conclude the proof of this step, it remains to show that

$$
\int_{\mathbf{C}_{i} \cap \Omega}\left|e\left(v_{i}\right)\right|^{p} d x \leq c \int_{C_{i} \cap \Omega}|e(u)|^{p} d x,
$$

for some $c=c(n, p, L)$. By (4.15), it is sufficient to show that $\int_{\cup_{q \in \mathcal{Q}_{g} q}}\left|e\left(\tilde{v}_{i}\right)\right|^{p} d x \leq$ $c \int_{C_{i} \cap \Omega}|e(u)|^{p} d x$. By the definition of $\tilde{v}_{i}$, one has

$$
e\left(\tilde{v}_{i}\right)=\sum_{q \in \mathcal{Q}_{g}}\left(e\left(w_{q}\right) \varphi_{q}+w_{q} \odot \nabla \varphi_{q}\right) .
$$

We need therefore to estimate the $L^{p}$ norm of $\sum_{q \in \mathcal{Q}_{g}} w_{q} \odot \nabla \varphi_{q}$ in terms of the $L^{p}$ norm of $e(u)$, since the other term in the sum satisfies the bound by (4.11). Notice that as $\sum_{q} \varphi_{q} \equiv 1$ in $\cup_{q \in \mathcal{Q}_{g}} q$, we have that $\sum_{q} \nabla \varphi_{q}=0$ in $\cup_{q \in \mathcal{Q}_{g}} q$ (where here and in what follows the sums run on cubes in $\mathcal{Q}_{g}$ ). Then, if we fix $q \in \mathcal{Q}_{g}$ and $x \in q^{\prime} \cap\left(\cup_{\hat{q} \in \mathcal{Q}_{g}} \hat{q}\right)$, we have

$$
\begin{aligned}
\sum_{\hat{q}} w_{\hat{q}}(x) \odot \nabla \varphi_{\hat{q}}(x) & =\sum_{\hat{q}} w_{\hat{q}}(x) \odot \nabla \varphi_{\hat{q}}(x)-w_{q}(x) \odot \sum_{\hat{q}} \nabla \varphi_{\hat{q}}(x) \\
& =\sum_{\hat{q}}\left(w_{\hat{q}}(x)-w_{q}(x)\right) \odot \nabla \varphi_{\hat{q}}(x) \\
& =\sum_{\hat{q}: q^{\prime} \cap \hat{q}^{\prime} \neq \emptyset}\left(w_{\hat{q}}(x)-w_{q}(x)\right) \odot \nabla \varphi_{\hat{q}}(x) .
\end{aligned}
$$

Note that the last equality in (4.18) follows since the only terms in the sum that have a non-zero contribution are the ones corresponding to cubes $\hat{q}$ such that $\hat{q}^{\prime}$ intersects $q^{\prime}$, whose number is bounded by $2^{n}$.

Now we observe that, if $q^{\prime} \cap \hat{q}^{\prime} \neq \varnothing$, then there are two cases: either $q$ and $\hat{q}$ are of the same size, or, alternatively, the edge length of one is twice the edge length of the other one. In either case

$$
\mathcal{L}^{n}\left(q^{\prime} \cap \hat{q}^{\prime}\right) \geq \beta_{1} \max \left\{\mathcal{L}^{n}\left(q^{\prime}\right), \mathcal{L}^{n}\left(\hat{q}^{\prime}\right)\right\}
$$

where $\beta_{1}=\beta_{1}(n)>0$ is an explicit constant depending only on the dimension. Now, to fix the ideas, assume that $q \in \mathcal{Q}_{k}$ and $\hat{q} \in \mathcal{Q}_{k+1}$; then, by Remark 3.4 and (4.12) (where we recall that $C=C(n, p)$ ), and since $q, \hat{q} \in \mathcal{Q}_{g}$,

$$
\begin{aligned}
\mathcal{L}^{n}\left(\omega_{q} \cup \omega_{\hat{q}}\right) & \leq C \delta 2^{-k}\left(\mathcal{H}^{n-1}\left(J_{u} \cap q^{\prime \prime}\right)+\mathcal{H}^{n-1}\left(J_{u} \cap \hat{q}^{\prime \prime}\right)\right) \\
& \leq c(n, p) \tau\left(\frac{\delta}{2^{k+1}}\right)^{n} \leq c(n, p) \tau \mathcal{L}^{n}\left(q^{\prime} \cap \hat{q}^{\prime}\right),
\end{aligned}
$$


where $c(n, p)$ denotes possibly different constants. Therefore, for every $q, \hat{q} \in \mathcal{Q}_{g}$ with $q^{\prime} \cap \hat{q}^{\prime} \neq \emptyset$,

$$
\mathcal{L}^{n}\left(\omega_{q} \cup \omega_{\hat{q}}\right) \leq c(n, p) \tau \mathcal{L}^{n}\left(q^{\prime} \cap \hat{q}^{\prime}\right)
$$

Hence, up to possibly reducing $\tau$, we have that

$$
\mathcal{L}^{n}\left(\left(q^{\prime} \cap \hat{q}^{\prime}\right) \backslash\left(\omega_{q} \cup \omega_{\hat{q}}\right)\right) \geq \beta_{2} \mathcal{L}^{n}\left(q^{\prime} \cap \hat{q}^{\prime}\right) \geq \beta_{1} \beta_{2} \max \left\{\mathcal{L}^{n}\left(q^{\prime}\right), \mathcal{L}^{n}\left(\hat{q}^{\prime}\right)\right\}
$$

for some $\beta_{2}>0$ depending on $n$ and $p$.

We now apply Lemma 4.4 to $w_{q}$ in $q^{\prime}$ and to $w_{\hat{q}}$ in $\hat{q}^{\prime}$, with $E=\left(q^{\prime} \cap \hat{q}^{\prime}\right) \backslash\left(\omega_{q} \cup \omega_{\hat{q}}\right)$ and $\alpha=\beta_{1} \beta_{2}$. Note that the constant $c$ in the lemma scales with the size of the domain; more precisely, for a dyadic cube $q^{\prime}$ with side length $\ell^{\prime}, c=c(n, \alpha, p)\left(\ell^{\prime}\right)^{p}$, with $c(n, \alpha, p)$ being the constant for the unit cube in $\mathbb{R}^{n}$. Then

$$
\begin{aligned}
& \int_{q^{\prime}}\left|w_{q}-a_{q}\right|^{p} d x \leq c\left(\ell^{\prime}\right)^{p} \int_{q^{\prime}}\left|e\left(w_{q}\right)\right|^{p} d x, \\
& \int_{\hat{q}^{\prime}}\left|w_{\hat{q}}-a_{\hat{q}}\right|^{p} d x \leq c\left(\hat{\ell}^{\prime}\right)^{p} \int_{\hat{q}^{\prime}}\left|e\left(w_{\hat{q}}\right)\right|^{p} d x,
\end{aligned}
$$

and

$$
a_{q}:=\arg \min _{a \in \mathcal{R}} \int_{E}\left|w_{q}-a\right|^{p} d x, \quad a_{\hat{q}}:=\arg \min _{a \in \mathcal{R}} \int_{E}\left|w_{\hat{q}}-a\right|^{p} d x .
$$

On the other hand, since $w_{q}=w_{\hat{q}}=u$ in $E$, we have that $a_{q}=a_{\hat{q}}=a$, and hence, thanks to (4.19)-(4.20) and (4.11),

$$
\begin{aligned}
\int_{q^{\prime} \cap \hat{q}^{\prime}}\left|w_{q}-w_{\hat{q}}\right|^{p} d x & \leq c(p) \int_{q^{\prime} \cap \hat{q}^{\prime}}\left|w_{q}-a\right|^{p} d x+c(p) \int_{q^{\prime} \cap \hat{q}^{\prime}}\left|w_{\hat{q}}-a\right|^{p} d x \\
& \leq c(n, p)\left(\left(\ell^{\prime}\right)^{p} \int_{q^{\prime}}\left|e\left(w_{q}\right)\right|^{p} d x+\left(\hat{\ell}^{\prime}\right)^{p} \int_{\hat{q}^{\prime}}\left|e\left(w_{\hat{q}}\right)\right|^{p} d x\right) \\
& \leq c(n, p) \ell^{p}\left(\int_{q^{\prime \prime}}|e(u)|^{p} d x+\int_{\hat{q}^{\prime \prime}}|e(u)|^{p} d x\right) .
\end{aligned}
$$

In conclusion, for a given $q \in \mathcal{Q}_{g}$, by (4.17), (4.18), (4.11) and the previous estimate,

$$
\begin{aligned}
& \int_{q}\left|e\left(\tilde{v}_{i}\right)\right|^{p} d x \leq c \sum_{\hat{q}: q^{\prime} \cap \hat{q}^{\prime} \neq \emptyset} \int_{q \cap \hat{q}^{\prime}}\left|e\left(w_{\hat{q}}\right)\right|^{p} d x \\
&+c \sum_{\hat{q}: q^{\prime} \cap \hat{q}^{\prime} \neq \emptyset}\left\|\nabla \varphi_{\hat{q}}\right\|_{L^{\infty}\left(\hat{q}^{\prime}\right)}^{p} \int_{q \cap \hat{q}^{\prime}}\left|w_{q}-w_{\hat{q}}\right|^{p} d x \\
& \leq c \sum_{\hat{q}: q^{\prime} \cap \hat{q}^{\prime} \neq \emptyset} \int_{\hat{q}^{\prime \prime}}|e(u)|^{p} d x,
\end{aligned}
$$


with $c=c(n, p)$, where we have used the fact that $\left\|\nabla \varphi_{\hat{q}}\right\|_{L^{\infty}\left(\hat{q}^{\prime}\right)} \leq c / \hat{\ell}$. Using that the cubes $q^{\prime \prime}$ have finite overlap, we have

$$
\int_{\cup_{q \in \mathcal{Q}_{g}} q}\left|e\left(\tilde{v}_{i}\right)\right|^{p} d x \leq c \int_{C_{i} \cap \Omega}|e(u)|^{p} d x,
$$

and, by (4.15) we obtain (4.16). We have then proved the estimates

$\int_{\mathbf{C}_{i} \cap \Omega}\left|e\left(v_{i}\right)\right|^{p} d x \leq c_{i} \int_{C_{i} \cap \Omega}|e(u)|^{p} d x, \quad \mathcal{H}^{n-1}\left(\partial^{*} \omega_{i}\right) \leq c_{i} \mathcal{H}^{n-1}\left(J_{u} \cap\left(C_{i} \cap \Omega\right)\right)$,

where $c_{i}=c_{i}(n, p, L)$ is the maximum of the two constants in (4.14) and (4.16).

Step 5: Conclusion. Recalling that $v=\sum_{i=0}^{N}\left(\phi_{i \mid C_{i} \cap \Omega}\right) v_{i}=\sum_{i=0}^{N}\left(\phi_{i \mid \mathbf{C}_{i} \cap \Omega}\right) v_{i}$ and $\omega=\bigcup_{i=0}^{N} \omega_{i}$ (see Step 3), and that the number of $C_{i}$ 's intersecting at every point of $\Omega$ is at most $\kappa+1$ (see Step 2), the statement holds true by setting

$$
c(n, p, \Omega):=(\kappa+1) \max \left\{c_{0}, c_{1}, \ldots, c_{N}, \tilde{c}\right\}
$$

where $\tilde{c}=\mathcal{H}^{n-1}\left(\partial^{*} \Omega\right) / \bar{c}$.

Note that, since $\mathcal{H}^{n-1}\left(\partial^{*} \Omega\right)$ can be estimated in terms of the parameters $N, r$ and $L$ introduced in Step 1, we have that $c(n, p, \Omega)=c(n, p, r, N, L)$.

An immediate consequence of Theorem 4.1 is the Korn's inequality below, whose proof is a direct adaptation of [16, Corollary 3.3].

Theorem 4.5 Under the same assumptions and notation of Theorem 4.1, and under the additional requirement that $\Omega$ is connected, there exists an affine function $a \in \mathcal{R}$ such that

$$
\int_{\Omega \backslash \omega}|\nabla u-\nabla a|^{p} d x \leq c(n, p, \Omega) \int_{\Omega}|e(u)|^{p} d x .
$$

Moreover,

$$
\left(\int_{\Omega \backslash \omega}|u-a|^{q} d x\right)^{\frac{1}{q}} \leq c(n, p, q, \Omega)\left(\int_{\Omega}|e(u)|^{p} d x\right)^{\frac{1}{p}},
$$

where $q \leq p^{*}$ if $p<n, q<\infty$ if $p=n$, and $q \leq \infty$ for $p>n$.

Proof Let $v \in W^{1, p}\left(\Omega ; \mathbb{R}^{n}\right)$ be given by Theorem 4.1. By Korn's inequality applied to $v$, there exists $A \in \mathbb{R}_{\mathrm{skw}}^{n \times n}$ such that

$$
\int_{\Omega}|\nabla v-A|^{p} d x \leq c(n, p, \Omega) \int_{\Omega}|e(v)|^{p} d x
$$


moreover, by applying Poincaré's inequality to the function $x \mapsto v(x)-A x$, there exists $b \in \mathbb{R}^{n}$ such that

$$
\int_{\Omega}|v(x)-A x-b|^{p} d x \leq c(n, p, \Omega) \int_{\Omega}|\nabla v-A|^{p} d x .
$$

We now define $a(x):=A x+b$; then $a \in \mathcal{R}$. Since $\nabla v=\nabla u \mathcal{L}^{n}$-a.e. on $\{v=u\}$, we have that

$$
\begin{aligned}
\int_{\Omega \backslash \omega}|\nabla u-\nabla a|^{p} d x & =\int_{\Omega \backslash \omega}|\nabla v-\nabla a|^{p} d x \\
& \leq c(n, p, \Omega) \int_{\Omega}|e(v)|^{p} d x \leq \tilde{c}(n, p, \Omega) \int_{\Omega}|e(u)|^{p} d x,
\end{aligned}
$$

where the last inequality follows by Theorem 4.1. This proves (4.21). Moreover, we can improve the norm on the left-hand side of (4.23) to the exponent $q$ of the Sobolev embedding of $W^{1, p}$ into $L^{q}$. Then, since $v=u$ in $\Omega \backslash \omega$, we have that

$$
\begin{aligned}
\int_{\Omega \backslash \omega}|u-a|^{q} d x & =\int_{\Omega \backslash \omega}|v-a|^{q} d x \\
& \leq c(n, p, q, \Omega) \int_{\Omega}|e(v)|^{p} d x \leq \tilde{c}(n, p, q, \Omega) \int_{\Omega}|e(u)|^{p} d x,
\end{aligned}
$$

which proves the estimate (4.22). Note that, if $p<n$, we can take $q=p^{*}$, and that if $p>n$ we can estimate $v-a$ in the Hölder seminorm $C^{0, \alpha}$, with $\alpha=1-\frac{n}{p}$.

\section{An approximation result}

In this last section, as an application, we show an approximation result in the spirit of [11, Theorem 3.1].

Theorem 5.1 Let $n \in \mathbb{N}$ with $n \geq 2, p \in(1, \infty)$, and let $\Omega \subset \mathbb{R}^{n}$ be a bounded open set of finite perimeter. Let $\varepsilon>0$. Then, for any $u \in G S B D^{p}(\Omega)$, there exist

- a closed set $\Gamma$, finite union of disjoint $(n-1)$-dimensional $C^{1}$ manifolds with $C^{1}$ boundary;

- a set $\tilde{\omega}$, finite union of cubes;

- a set of finite perimeter $\hat{\omega}$;

such that

$$
\mathcal{H}^{n-1}\left(J_{u} \triangle \Gamma\right)+\mathcal{H}^{n-1}\left(\partial^{*} \tilde{\omega}\right)+\mathcal{H}^{n-1}\left(\partial^{*} \hat{\omega}\right)<\varepsilon
$$

Moreover, there exists a function $w \in G S B D^{p}(\Omega) \cap W^{1, p}\left(\Omega \backslash(\Gamma \cup \overline{\tilde{\omega}}) ; \mathbb{R}^{n}\right)$ such that $\{w \neq u\} \subset \tilde{\omega} \cup \hat{\omega}$,

$$
\int_{\Omega \backslash \tilde{\omega}}|e(w)|^{p} d x \leq(1+\varepsilon) \int_{\Omega}|e(u)|^{p} d x
$$


and

$$
\mathcal{H}^{n-1}\left(\Gamma \cap\left\{w^{ \pm} \neq u^{ \pm}\right\}\right)<\varepsilon
$$

where $w^{ \pm}$and $u^{ \pm}$denote the traces of $w$ and $u$ on the two sides of $\Gamma$.

Corollary 5.2 Under the same assumptions and notation of Theorem 5.1, for $u \in$ $G S B D^{p}(\Omega)$ the approximate gradient $\nabla$ u exists $\mathcal{L}^{n}$-a.e. in $\Omega$.

Proof of Corollary 5.2 Let $k \in \mathbb{N}$, and let $\tilde{\omega}_{k}, \hat{\omega}_{k}$ and $w_{k}$ be as in Theorem 5.1, for $\varepsilon=\frac{1}{k}$. Since $w_{k} \in G S B D^{p}(\Omega) \cap W^{1, p}\left(\Omega \backslash\left(\Gamma \cup \overline{\tilde{\omega}_{k}}\right) ; \mathbb{R}^{n}\right)$, we have in particular that $\nabla w_{k}$ exists $\mathcal{L}^{n}$-a.e. in $\Omega \backslash \tilde{\omega}_{k}$. Moreover, as $u=w_{k}$ in $\Omega \backslash\left(\tilde{\omega}_{k} \cup \hat{\omega}_{k}\right)$, it follows that $\nabla u$ exists $\mathcal{L}^{n}$-a.e. in $\Omega \backslash\left(\tilde{\omega}_{k} \cup \hat{\omega}_{k}\right)$ (note that $\tilde{\omega}_{k}$ is a finite union of cubes, and hence its boundary is $\mathcal{L}^{n}$-negligible). By repeating this argument for every $k \in \mathbb{N}$ we have that $\nabla u$ exists $\mathcal{L}^{n}$-a.e. in $\Omega \backslash \omega$, where

$$
\omega:=\bigcap_{k \in \mathbb{N}}\left(\tilde{\omega}_{k} \cup \hat{\omega}_{k}\right) .
$$

Since by (5.1) and Remark 3.4 we have that $\mathcal{L}^{n}\left(\tilde{\omega}_{k} \cup \hat{\omega}_{k}\right) \leq C\left(\frac{1}{k}\right)^{n /(n-1)}$ for every $k \in \mathbb{N}$, where $C=C(n)$, it follows that $\mathcal{L}^{n}(\omega)=0$. Hence we can conclude that $\nabla u$ exists $\mathcal{L}^{n}$-a.e. in $\Omega$.

Note that in the case $p=2$ the result in Corollary 5.2 has been obtained in [28], as a consequence of the embedding $G S B D^{2}(\Omega) \subset(G B V(\Omega))^{n}$ (see [28, Theorem 2.9]), for $n \geq 2$.

Theorem 5.1 will follow as a special case of the following technical proposition.

Proposition 5.3 Let $n \in \mathbb{N}$ with $n \geq 2$, and $p \in(1, \infty)$. Let $u \in G S B D^{p}\left(\mathbb{R}^{n}\right)$ and let $J$ be a countably $\left(\mathcal{H}^{n-1}, n-1\right)$ rectifiable set with $J_{u} \subset J$ and $\mathcal{H}^{n-1}(J)<+\infty$. Let $\varepsilon>0$. Then there exist

- a closed set $\Gamma$, finite union of disjoint $(n-1)$-dimensional $C^{1}$ manifolds with $C^{1}$ boundary;

- a set $\tilde{\omega}$, finite union of cubes;

- a set of finite perimeter $\hat{\omega}$;

- a function $w \in G S B D^{p}\left(\mathbb{R}^{n}\right)$, with $w \in W^{1, p}\left(B_{R}(0) \backslash(\Gamma \cup \overline{\tilde{\omega}}) ; \mathbb{R}^{n}\right)$ for any $R>0$; such that $w=u \mathcal{L}^{n}$-a.e. in $\mathbb{R}^{n} \backslash(\tilde{\omega} \cup \hat{\omega})$, and

$$
\begin{aligned}
& \mathcal{H}^{n-1}(J \triangle \Gamma) \leq \varepsilon, \\
& \int_{\mathbb{R}^{n} \backslash \tilde{\omega}}|e(w)|^{p} d x \leq(1+\varepsilon) \int_{\mathbb{R}^{n}}|e(u)|^{p} d x, \\
& \mathcal{H}^{n-1}\left(\partial^{*} \tilde{\omega}\right)+\mathcal{H}^{n-1}\left(\partial^{*} \hat{\omega}\right) \leq \varepsilon .
\end{aligned}
$$

Moreover, $u^{ \pm}(x)=w^{ \pm}(x)$ for $\mathcal{H}^{n-1}$-a.e. $x \in \Gamma \backslash\left(\overline{\tilde{\omega}} \cup \hat{\omega}^{(1)} \cup \partial^{*} \hat{\omega}\right)$, and $\mathcal{H}^{n-1}(\Gamma \cap$ $\left.\left\{w^{ \pm} \neq u^{ \pm}\right\}\right)<\varepsilon$, where we used (h) in Sect. 2. 
We recall that, for $u \in G S B D\left(\mathbb{R}^{n}\right)$, the set $J_{u}$ is countably $\left(\mathcal{H}^{n-1}, n-1\right)$ rectifiable [22, Section 6] (see [25, Section 3.2.14] for the definition), so that the assumption $J_{u} \subset J$ is not restrictive.

Theorem 5.1 is deduced from Proposition 5.3 in the following way. Let $\Omega \subset \mathbb{R}^{n}$ and $u \in G S B D^{p}(\Omega)$ as in the assumptions of Theorem 5.1, and let $\tilde{u}$ denote the extension of $u$ to $\mathbb{R}^{n}$ obtained by setting $\tilde{u}:=0$ outside $\Omega$. Then $\tilde{u} \in G S B D^{p}\left(\mathbb{R}^{n}\right)$, and by applying Proposition 5.3 to $\tilde{u}$ and $J=J_{\tilde{u}}$ we obtain the claim.

Proof of Proposition 5.3 Let $u, J$ and $\varepsilon$ be as in the statement, and let $\rho>0$ and $\alpha>0$ be constants to be determined later. We split the proof into several steps.

Step 1: Covering the jump set Since $J$ is countably $\left(\mathcal{H}^{n-1}, n-1\right)$ rectifiable and $\mathcal{H}^{n-1}(J)<+\infty$, by [25, Theorem 3.2.29] there exists a countable family $\left(M_{k}\right)_{k \in \mathbb{N}}$ of $C^{1}$ hypersurfaces such that

$$
\mathcal{H}^{n-1}\left(J \backslash \bigcup_{k=1}^{\infty} M_{k}\right)=0
$$

With no loss of generality we can assume that for each $k \in \mathbb{N}$ the manifold $M_{k}$ is a Lipschitz graph with Lipschitz constant less than 1/4 [2, Theorem 2.76]. Then, for every $k \in \mathbb{N}, \mathcal{H}^{n-1}$-a.e. point in $J \cap M_{k}$ is a point of $\mathcal{H}^{n-1}$-density 1 both for $J$ and $J \cap M_{k}$, namely

$$
\lim _{r \rightarrow 0^{+}} \frac{\mathcal{H}^{n-1}\left(J \cap \bar{B}_{r}(x)\right)}{\gamma_{n-1} r^{n-1}}=\lim _{r \rightarrow 0^{+}} \frac{\mathcal{H}^{n-1}\left(\left(J \cap M_{k}\right) \cap \bar{B}_{r}(x)\right)}{\gamma_{n-1} r^{n-1}}=1,
$$

for every $k \in \mathbb{N}$ and $\mathcal{H}^{n-1}$-a.e. $x \in J \cap M_{k}$. From this, it follows that for every $k \in \mathbb{N}$ and for $\mathcal{H}^{n-1}$-a.e. $x \in J \cap M_{k}$ there exists $\eta(\alpha, x) \in(0, \rho)$ such that

$$
\begin{aligned}
& \left|\mathcal{H}^{n-1}\left(\bar{B}_{r}(x) \cap J\right)-\gamma_{n-1} r^{n-1}\right| \leq \alpha \gamma_{n-1} r^{n-1}, \\
& \left|\mathcal{H}^{n-1}\left(\bar{B}_{r}(x) \cap\left(J \cap M_{k}\right)\right)-\gamma_{n-1} r^{n-1}\right| \leq \alpha \gamma_{n-1} r^{n-1},
\end{aligned}
$$

and

$$
\mathcal{H}^{n-1}\left(\bar{B}_{r}(x) \cap\left(J \Delta M_{k}\right)\right) \leq \alpha \mathcal{H}^{n-1}\left(\bar{B}_{r}(x) \cap J\right),
$$

for every $r \leq \eta(\alpha, x)$. In other words, up to sufficiently restricting the radius of the ball, we can assume that the main content of $J$ in a ball centred at a point $x \in J \cap M_{k}$ comes from $M_{k}$, and not from the other components $M_{j}$, for $j \neq k$.

Let $M:=J \cap \cup_{k} M_{k}$. Note that the family $\left\{\bar{B}_{r}(x): x \in M, r \leq \eta(\alpha, x)\right\}$ is a fine cover of $M$ (see [2, Section 2.4]). Then, applying the Vitali-Besicovitch's Covering Theorem [2, Theorem 2.19] to $A=M$ and $\mu=\mathcal{H}^{n-1}\llcorner M$, there exists a disjoint subfamily $\left\{\bar{B}_{r(\alpha, x)}(x): x \in M^{\prime}\right\}$, for some $M^{\prime} \subset M$ and $r(\alpha, x) \leq \eta(\alpha, x)$, such that

$$
\mathcal{H}^{n-1}\left(J \backslash \bigcup_{x \in M^{\prime}} \bar{B}_{r(\alpha, x)}(x)\right)=0 \text {. }
$$


Moreover, the subfamily above is countable, since it is composed of disjoint sets with nonempty interior. Hence, there exists a sequence $\left\{x_{i}\right\}_{i \in \mathbb{N}} \subset \cup_{k} M_{k}$ such that

$$
\mathcal{H}^{n-1}\left(J \backslash \bigcup_{i \in \mathbb{N}} \bar{B}_{i}\right)=0
$$

where $B_{i}:=B_{r_{i}}\left(x_{i}\right)$ for every $i \in \mathbb{N}$, and where we set $r_{i}:=r\left(\alpha, x_{i}\right)$. Finally, note that from the identity above it follows that there exists $N=N(\alpha) \in \mathbb{N}$ such that

$$
\mathcal{H}^{n-1}\left(J \backslash \bigcup_{i=1}^{N} \bar{B}_{i}\right)<\alpha
$$

Given $i \in\{1, \ldots, N\}$, let $k(i) \in \mathbb{N}$ be such that $x_{i} \in M_{k(i)}$ and define $\Gamma_{i}:=M_{k(i)} \cap \bar{B}_{i}$. Then $B_{i} \backslash \Gamma_{i}$ has two (Lipschitz) connected components, and the following properties are satisfied:

a) $\Gamma_{i}$ is a Lipschitz graph with constant less than $1 / 4$;

b) $\left|\mathcal{H}^{n-1}\left(\bar{B}_{r}\left(x_{i}\right) \cap J\right)-\gamma_{n-1} r^{n-1}\right| \leq \alpha \gamma_{n-1} r^{n-1}$ for all $r \leq r_{i}$;

c) $\mathcal{H}^{n-1}\left(\bar{B}_{i} \cap\left(J \triangle \Gamma_{i}\right)\right) \leq \alpha \mathcal{H}^{n-1}\left(\bar{B}_{i} \cap J\right)$;

d) $\mathcal{H}^{n-1}\left(J \backslash \bigcup_{i=1}^{N} \Gamma_{i}\right) \leq \alpha\left(1+\mathcal{H}^{n-1}(J)\right)$;

e) $\mathcal{L}^{n}\left(\bigcup_{i=1}^{N} \bar{B}_{i}\right) \leq \frac{\gamma_{n}}{\gamma_{n-1}} \frac{\rho}{1-\alpha} \mathcal{H}^{n-1}(J)$.

Properties a), b), c) follow immediately. We now prove property d). First, note that

$$
J \backslash \bigcup_{i=1}^{N} \Gamma_{i}=\left(J \backslash \bigcup_{i=1}^{N} \bar{B}_{i}\right) \cup\left(\bigcup_{i=1}^{N} J \cap\left(\bar{B}_{i} \backslash \Gamma_{i}\right)\right) .
$$

Hence, by (5.2) and by property c)

$$
\begin{aligned}
\mathcal{H}^{n-1}\left(J \backslash \bigcup_{i=1}^{N} \Gamma_{i}\right) & <\alpha+\mathcal{H}^{n-1}\left(\bigcup_{i=1}^{N} J \cap\left(\bar{B}_{i} \backslash \Gamma_{i}\right)\right) \\
& \leq \alpha+\sum_{i=1}^{N} \mathcal{H}^{n-1}\left(J \cap\left(\bar{B}_{i} \backslash \Gamma_{i}\right)\right) \leq \alpha\left(1+\mathcal{H}^{n-1}(J)\right),
\end{aligned}
$$

which shows d). To see e) note that, since the closed balls are disjoint,

$$
\begin{aligned}
\mathcal{L}^{n}\left(\bigcup_{i=1}^{N} B_{i}\right) & =\sum_{i=1}^{N} \gamma_{n} r_{i}^{n} \leq \frac{\gamma_{n} \rho}{\gamma_{n-1}} \sum_{i=1}^{N} \gamma_{n-1} r_{i}^{n-1} \\
& \leq \frac{\gamma_{n}}{\gamma_{n-1}} \frac{\rho}{1-\alpha} \sum_{i=1}^{N} \mathcal{H}^{n-1}\left(\bar{B}_{i} \cap J\right) \leq \frac{\gamma_{n}}{\gamma_{n-1}} \frac{\rho}{1-\alpha} \mathcal{H}^{n-1}(J),
\end{aligned}
$$


where we have also used $b$ ).

Finally, letting $\Gamma:=\bigcup_{i=1}^{N} \Gamma_{i}$, one has that $\Gamma$ is a finite union of disjoint $C^{1}$ manifolds with $C^{1}$ boundary. Moreover, thanks to c) and d),

$$
\mathcal{H}^{n-1}(J \triangle \Gamma) \leq \alpha\left(1+2 \mathcal{H}^{n-1}(J)\right)
$$

Step 2: Cleaning the jump set in the balls $B_{i}$. We split this step into further substeps. Step 2.1: Application of Theorem 4.1 in the balls $B_{i}$. Let us denote $B_{i}^{+}, B_{i}^{-}$the connected components of $B_{i} \backslash \Gamma_{i}$. Thanks to Theorem 4.1, in each $B_{i}^{ \pm}, i=1, \ldots, N$, there exists a set of finite perimeter $\omega_{i}^{ \pm}$and a function $v_{i}^{ \pm} \in W^{1, p}\left(B_{i}^{ \pm} ; \mathbb{R}^{n}\right)$ such that

$$
\begin{gathered}
v_{i}^{ \pm}=u \quad \text { in } B_{i}^{ \pm} \backslash \omega_{i}^{ \pm}, \\
\int_{B_{i}^{ \pm}}\left|e\left(v_{i}^{ \pm}\right)\right|^{p} d x \leq c_{i}^{ \pm} \int_{B_{i}^{ \pm}}|e(u)|^{p} d x \\
\mathcal{H}^{n-1}\left(\partial^{*} \omega_{i}^{ \pm}\right) \leq c_{i}^{ \pm} \mathcal{H}^{n-1}\left(J \cap B_{i}^{ \pm}\right),
\end{gathered}
$$

where $c_{i}^{ \pm}=c_{i}^{ \pm}(n, p)$.

Step 2.2: The constant $c:=\max \left\{c_{i}^{ \pm}: i=1, \ldots, N\right\}$ is bounded uniformly in $N$ (and hence in $\alpha$ ). First we note that, due to the invariance of $c_{i}^{ \pm}$under uniform rescalings of the domain, it is not restrictive to assume that $B_{i}$ has unit radius for every $i=1, \ldots, N$.

Now, let $i=1, \ldots, N$ be fixed, and consider for instance the set $B_{i}^{+}$. Since $B_{i}^{+}$ is a Lipschitz set, there exist $\hat{r}_{i}^{+}$and $\hat{L}_{i}^{+}$such that for every point of $\partial B_{i}^{+}$we can construct a cylinder with radius $2 \hat{r}_{i}^{+}$and half-height $4 \hat{L}_{i}^{+} \hat{r}_{i}^{+}$where $\partial B_{i}^{+}$is the graph of an $\hat{L}_{i}^{+}$-Lipschitz function. Since $\Gamma_{i}$ is a Lipschitz graph with constant less than $1 / 4$, a careful construction shows that one can find $\hat{r}_{i}^{+}=\hat{r}$ and $\hat{L}_{i}^{+}=\hat{L}$ independent of the particular manifold $\Gamma_{i}$. Finally, let $\hat{N}_{i}^{+} \in \mathbb{N}$ be given by point (ii) of Remark 4.3, for the domain $B_{i}^{+}$. By the same remark it follows that $\hat{N}_{i}^{+}$satisfies the estimates

$$
\left\{\begin{array}{l}
\hat{N}_{i}^{+} \hat{r}^{n-1} \leq\left(5^{n-1} / \gamma_{n-1}\right) \mathcal{H}^{n-1}\left(\partial B_{i}^{+}\right) \leq\left(5^{n-1} / \gamma_{n-1}\right) C_{2}(n), \\
C_{1}(n) \leq \mathcal{H}^{n-1}\left(\partial B_{i}^{+}\right) \leq \hat{N}_{i}^{+} \gamma_{n-1} \hat{r}^{n-1} \sqrt{1+\hat{L}^{2}}
\end{array}\right.
$$

where we have used that

$$
\begin{aligned}
C_{1}(n):= & \mathcal{H}^{n-1}\left(\mathbb{S}^{n-1} \cap\left\{x_{n} \geq 1 / 4\right\}\right) \leq \mathcal{H}^{n-1}\left(\partial B_{i}^{+}\right) \\
& \leq \mathcal{H}^{n-1}\left(\mathbb{S}^{n-1}\right)+\mathcal{H}^{n-1}\left(\Gamma_{i}\right) \leq n \gamma_{n}+\gamma_{n-1} \sqrt{1+(1 / 4)^{2}}=: C_{2}(n) .
\end{aligned}
$$

From this it follows that $\hat{N}_{i}^{+}$can be chosen to be depending only on $n$.

In conclusion, $\left(\hat{N}_{i}^{+}, \hat{r}_{i}^{+}, \hat{L}_{i}^{+}\right)$can be chosen uniformly in $i$. Since, by Remark 4.3, the constant $c_{i}^{+}$depends on $B_{i}^{+}$only via $\left(\hat{N}_{i}^{+}, \hat{r}_{i}^{+}, \hat{L}_{i}^{+}\right)$, we finally conclude that the constant $c:=\max \left\{c_{i}^{ \pm}: i=1, \ldots, N\right\}$ can be bounded uniformly in $N$, and hence, as $N=N(\alpha)$, uniformly in $\alpha$. In particular, in (5.4)-(5.6), we can replace $c_{i}^{ \pm}$with the uniform constant $c$. 
Step 2.3: Conclusion. Thanks to b) and c) in Step 1, we have that $\mathcal{H}^{n-1}\left(J \cap B_{i}^{ \pm}\right) \leq \mathcal{H}^{n-1}\left(\bar{B}_{i} \cap\left(J \triangle \Gamma_{i}\right)\right) \leq \alpha \mathcal{H}^{n-1}\left(\bar{B}_{i} \cap J\right) \leq \alpha(1+\alpha) \gamma_{n-1} r_{i}^{n-1}$,

and hence from (5.6) and Step 2.2, $\mathcal{H}^{n-1}\left(\partial^{*} \omega_{i}^{ \pm}\right) \leq c \alpha(1+\alpha) \gamma_{n-1} r_{i}^{n-1}$. (Note that, in the case where $\omega_{i}^{ \pm}=B_{i}^{ \pm}$, we can simply let $v_{i}^{ \pm}=0$; however by choosing $\alpha>0$ small enough we can assume with no loss of generality that this does not happen.)

It follows that on $\partial B_{i}$ the trace of each $v_{i}^{ \pm}$coincides with the trace of $u$, except on a set of total measure at most $2 c \alpha(1+\alpha) \gamma_{n-1} r_{i}^{n-1}$. Let now

$$
v(x):= \begin{cases}v_{i}^{ \pm}(x) & \text { if } x \in B_{i}^{ \pm}, i=1, \ldots, N \\ u(x) & \text { if } x \in \mathbb{R}^{n} \backslash \bigcup_{i=1}^{N} B_{i} .\end{cases}
$$

Then $v \in G S B D^{p}\left(\mathbb{R}^{n}\right)$, and we have the following properties:

1) $J_{v} \cap B_{i} \subset \Gamma_{i}$ for each $i=1, \ldots, N$;

2) $\sum_{i=1}^{N} \mathcal{H}^{n-1}\left(J_{v} \cap \partial B_{i}\right) \leq \alpha(1+2 c) \mathcal{H}^{n-1}(J)$;

3) $\mathcal{H}^{n-1}\left(J_{v} \backslash \Gamma\right) \leq \alpha\left(1+(1+2 c) \mathcal{H}^{n-1}(J)\right)$.

Property 1) follows from the definition of $v$. For property 2), note that by c) and (5.6)

$$
\begin{aligned}
\sum_{i=1}^{N} \mathcal{H}^{n-1}\left(J_{v} \cap \partial B_{i}\right) \leq & \sum_{i=1}^{N} \mathcal{H}^{n-1}\left(J_{u} \cap \partial B_{i}\right)+\sum_{i=1}^{N} \mathcal{H}^{n-1}\left(\partial B_{i} \cap\left(\partial^{*} \omega_{i}^{+} \cup \partial^{*} \omega_{i}^{-}\right)\right) \\
& \leq \sum_{i=1}^{N} \mathcal{H}^{n-1}\left(J \cap\left(\bar{B}_{i} \backslash \Gamma_{i}\right)\right)+\sum_{i=1}^{N}\left(\mathcal{H}^{n-1}\left(\partial^{*} \omega_{i}^{+}\right)+\mathcal{H}^{n-1}\left(\partial^{*} \omega_{i}^{-}\right)\right) \\
& \leq \alpha \mathcal{H}^{n-1}(J)+\sum_{i=1}^{N}\left(\mathcal{H}^{n-1}\left(\partial^{*} \omega_{i}^{+}\right)+\mathcal{H}^{n-1}\left(\partial^{*} \omega_{i}^{-}\right)\right) \\
& \leq \alpha \mathcal{H}^{n-1}(J)+2 c \sum_{i=1}^{N}\left(\mathcal{H}^{n-1}\left(J \cap B_{i}^{+}\right)+\mathcal{H}^{n-1}\left(J \cap B_{i}^{-}\right)\right) \\
& \leq \alpha \mathcal{H}^{n-1}(J)+2 c \sum_{i=1}^{N}\left(\mathcal{H}^{n-1}\left(\left(J \cap B_{i}\right) \backslash \Gamma_{i}\right)\right) \\
& \leq \alpha(1+2 c) \mathcal{H}^{n-1}(J) .
\end{aligned}
$$

Let us show property 3). By $(5.2), 1)$ and 2) we have that

$$
\begin{aligned}
\mathcal{H}^{n-1}\left(J_{v} \backslash \Gamma\right) & \leq \mathcal{H}^{n-1}\left(J \backslash \bigcup_{i=1}^{N} \bar{B}_{i}\right)+\sum_{i=1}^{N} \mathcal{H}^{n-1}\left(J_{v} \cap \partial B_{i}\right) \\
& \leq \alpha\left(1+(1+2 c) \mathcal{H}^{n-1}(J)\right) .
\end{aligned}
$$


Moreover, letting $\omega_{B}:=\bigcup_{i=1}^{N}\left(\omega_{i}^{+} \cup \omega_{i}^{-}\right)$, one has that $v=u \mathcal{L}^{n}$-a.e. in $\mathbb{R}^{n} \backslash \omega_{B}$ and, by $(5.6), \mathcal{H}^{n-1}\left(\partial^{*} \omega_{B}\right) \leq 2 c \alpha \mathcal{H}^{n-1}(J)$.

Step 3: Cleaning the jump set in the rest of the domain. We now pick $\delta>0$ with

$$
0<\delta \leq 0.8 \alpha\left(\min _{i=1, \ldots, N} r_{i}\right) /(2 \sqrt{n})
$$

and consider the covering of $\mathbb{R}^{n} \backslash \bigcup_{i=1}^{N} B_{i}$ made of:

- the family $\mathcal{Q}_{1}$ of cubes $\delta z+[0, \delta]^{n}, z \in \mathbb{Z}^{n}$, which intersect $\mathbb{R}^{n} \backslash \bigcup_{i=1}^{N} B_{i}$;

- the family $\mathcal{Q}_{2}$ of cubes $\delta z+[0, \delta]^{n}, z \in \mathbb{Z}^{n}$, which are not in $\mathcal{Q}_{1}$, but intersect some cubes in $\mathcal{Q}_{1}$.

We set $\mathcal{Q}=\mathcal{Q}_{1} \cup \mathcal{Q}_{2}$. For each $q \in \mathcal{Q}$, we denote with $q \subset q^{\prime} \subset q^{\prime \prime}$ the concentric cubes $q^{\prime}$ and $q^{\prime \prime}$ with edges $(9 / 8) \delta$ and $(10 / 8) \delta$, respectively; we also denote with $\ell<\ell^{\prime}<\ell^{\prime \prime}$ the lengths of the edges of $q, q^{\prime}$ and $q^{\prime \prime}$, respectively, so that in particular, $\ell^{\prime}=(1-0.1) \ell^{\prime \prime}$. For each $i$, letting $B_{i}^{\prime}:=B_{(1-\alpha) r_{i}}\left(x_{i}\right)$, we observe that, since $\Gamma_{i}$ are equi-Lipschitz with constant less than $\frac{1}{2}$, one has

$$
\mathcal{H}^{n-1}\left(\Gamma_{i} \cap\left(B_{i} \backslash B_{i}^{\prime}\right)\right) \leq c \alpha r_{i}^{n-1} \leq c \alpha \mathcal{H}^{n-1}\left(J \cap \bar{B}_{i}\right)
$$

for some constant $c=c(n)$, where in the last inequality we used property b). Hence, since by the definition of $\delta$ we have that $q^{\prime \prime} \cap B_{i}^{\prime}=\emptyset$ for each $q \in \mathcal{Q}$ and for every $i$, we have that

$$
J_{v} \cap \bigcup_{q \in \mathcal{Q}} q^{\prime \prime}=\left(J_{v} \backslash\left(\bigcup_{i=1}^{N} \bar{B}_{i}\right)\right) \cup\left(\bigcup_{i=1}^{N} J_{v} \cap\left(B_{i} \backslash B_{i}^{\prime}\right)\right) \cup\left(\bigcup_{i=1}^{N}\left(J_{v} \cap \partial B_{i}\right)\right)
$$

Then, recalling 1), and using (5.2), (5.7) and 2), we have

$$
\mathcal{H}^{n-1}\left(J_{v} \cap \bigcup_{q \in \mathcal{Q}} q^{\prime \prime}\right) \leq \alpha\left(1+(1+3 c) \mathcal{H}^{n-1}(J)\right)
$$

for a constant $c$ depending only on the dimension.

We now invoke Theorem 3.2 (in its version for cubes, as noted in Remark 3.6) for parameters $\varepsilon=1$ (which thus needs not be the $\varepsilon$ of the statement), and $\sigma=0.1$, and find constants $C=C(n, p)$ and $\tau=\tau(n, p)$ satisfying the thesis of the theorem.

Let $\mathcal{Q}_{g} \subset \mathcal{Q}$ denote the set of cubes $q$ such that $\mathcal{H}^{n-1}\left(J_{v} \cap q^{\prime \prime}\right) \leq \tau \delta^{n-1}$, let $\mathcal{Q}_{b}:=\mathcal{Q} \backslash \mathcal{Q}_{g}$, and $\tilde{\omega}:=\bigcup_{q \in \mathcal{Q}_{b}} q$. Since for $q \in \mathcal{Q}_{b}$ one has $\mathcal{H}^{n-1}\left(J_{v} \cap q^{\prime \prime}\right)>\tau \delta^{n-1}$, there can be only a finite number of such cubes. Moreover, thanks to (5.8) we have that

$$
\left\{\begin{array}{l}
\mathcal{H}^{n-1}\left(\partial^{*} \tilde{\omega}\right) \leq \frac{c(n)}{\tau} \alpha\left(1+(1+3 c) \mathcal{H}^{n-1}(J)\right) \\
\mathcal{L}^{n}(\tilde{\omega}) \leq \frac{c(n)}{\tau} \delta \alpha\left(1+(1+3 c) \mathcal{H}^{n-1}(J)\right)
\end{array}\right.
$$


Now, let $q \in \mathcal{Q}_{g}$. By Theorem 3.2 there exist $w_{q} \in G S B D^{p}\left(q^{\prime \prime}\right) \cap W^{1, p}\left(q^{\prime} ; \mathbb{R}^{n}\right)$ and $\omega_{q} \subset q^{\prime \prime}$, with $w_{q}=v$ in $q^{\prime \prime} \backslash \omega_{q}$, and

$$
\begin{aligned}
& \int_{q^{\prime \prime}}\left|e\left(w_{q}\right)\right|^{p} d x \leq 2 \int_{q^{\prime \prime}}|e(v)|^{p} d x, \\
& \int_{\omega_{q}}\left|e\left(w_{q}\right)\right|^{p} d x \leq 2 \int_{\omega_{q}}|e(v)|^{p} d x, \\
& \mathcal{H}^{n-1}\left(\partial^{*} \omega_{q}\right) \leq C \mathcal{H}^{n-1}\left(J_{v} \cap q^{\prime \prime}\right),
\end{aligned}
$$

where (5.10) follows by Remark 3.5.

Possibly reducing $\tau$, we may assume that if $q^{\prime} \cap \Gamma_{i} \neq \varnothing$ for some $i=1, \ldots, N$, then $\mathcal{H}^{n-1}\left(\Gamma_{i} \cap q^{\prime \prime}\right) \geq \tau \delta^{n-1}$ (see point a) in Step 1), so that $q \notin \mathcal{Q}_{g}$. It then follows that for any $q \in \mathcal{Q}_{g}$, when $q^{\prime} \subset B_{i}$ for some $i$ (or more precisely $q^{\prime} \subset B_{i}^{ \pm}$, since $q \in \mathcal{Q}_{g}$ is such that $q^{\prime}$ does not intersect $\Gamma_{i}$ ), then $w_{q}=v$ in $q^{\prime}$.

We now 'glue' the functions $w_{q}$ in order to find a global $W_{\text {loc }}^{1, p}$ function as in the claim of the theorem. To do so, we introduce a cut-off function $\psi \in$ $C_{c}^{\infty}\left((-9 / 16,9 / 16)^{n} ;[0,1]\right)$ with $\eta=1$ on $[-1 / 2,1 / 2]^{n}$. Then for each $q \in \mathcal{Q}_{g}$, with center $c_{q}$, we define $\psi_{q}(x):=\psi\left(\left(x-c_{q}\right) / \delta\right) \in C_{c}^{\infty}\left(q^{\prime} ;[0,1]\right)$, so that $\psi_{q}=1$ on $q$. We then let, for $x \in G:=\bigcup_{q \in \mathcal{Q}_{g}} q, \varphi_{q}(x):=\psi_{q}(x) /\left(\sum_{\hat{q} \in \mathcal{Q}_{g}} \psi_{\hat{q}}(x)\right) \in[0,1]$, and

$$
w(x):= \begin{cases}\sum_{q \in \mathcal{Q}_{g}} w_{q}(x) \varphi_{q}(x) & \text { if } x \in G, \\ 0 & \text { if } x \in \tilde{\omega}, \\ v(x) & \text { if } x \in \mathbb{R}^{n} \backslash(G \cup \tilde{\omega}) .\end{cases}
$$

By construction we have that $w \in W^{1, p}\left(B_{R}(0) \backslash(\Gamma \cup \overline{\tilde{\omega}}) ; \mathbb{R}^{n}\right)$ for any $R>0$. Indeed, we observe that $\mathbb{R}^{n} \backslash(G \cup \tilde{\omega}) \subset \bigcup_{i} B_{i}$, and hence (by the definition of $v$ ), in this set the function $w$ is Sobolev outside $\Gamma$. Moreover, $w$ does not jump on the intersection between the boundaries of $G$ and $\mathbb{R}^{n} \backslash(G \cup \tilde{\omega})$. Indeed, if $q \in \mathcal{Q}_{g}$ is any cube touching the set $\mathbb{R}^{n} \backslash(G \cup \tilde{\omega})$, then it has to be that $q \in \mathcal{Q}_{2}$ and $q \subset B_{i}$ for some $i$ (and therefore, as observed before, $w_{q}=v$ in $q^{\prime}$ ).

Let $\omega_{G}:=\bigcup_{q \in \mathcal{Q}_{g}} \omega_{q}$ and $\hat{\omega}:=\omega_{B} \cup \omega_{G}$. Then, $w=u$ in $\mathbb{R}^{n} \backslash(\tilde{\omega} \cup \hat{\omega})$, since $w=v$ outside $\omega_{G} \cup \tilde{\omega}$, and $v=u$ outside $\omega_{B}$. Hence $e(w)=e(u)$ in that set.

Step 3.1: Traces of $w$ on $\Gamma$. We now compare the traces of $w$ and of $u$ on the two sides of $\Gamma$. We have already observed that $w=u$ in $\mathbb{R}^{n} \backslash(\tilde{\omega} \cup \hat{\omega})$, where $\hat{\omega}=\omega_{B} \cup \omega_{G}$.

Note that, since $q^{\prime \prime} \cap B_{i}^{\prime}=\emptyset$ for every $q \in \mathcal{Q}$ and for every $i=1, \ldots, N$, $\left(\overline{\tilde{\omega}} \cup \overline{\omega_{G}}\right) \cap \bigcup_{i} B_{i}^{\prime}=\emptyset$. Hence the exceptional sets $\tilde{\omega}$ and $\omega_{G}$ affect the traces of $w$ only on a subset of $\Gamma$ of small (in terms of $\alpha$ ) $\mathcal{H}^{n-1}$-measure, by (5.7), namely

$$
\mathcal{H}^{n-1}\left(\Gamma \cap\left\{w^{ \pm} \neq v^{ \pm}\right\}\right) \leq c \alpha \mathcal{H}^{n-1}(J)
$$

For the set $\omega_{B}$ we observe that, by the definition of $v$ and by (5.4), for every $i=$ $1, \ldots, N, v^{ \pm}(x)=u^{ \pm}(x)$ for $\mathcal{H}^{n-1}$-a.e. $x \in \Gamma_{i} \backslash\left(\partial^{*} \omega_{i}^{+} \cup \partial^{*} \omega_{i}^{-}\right)$. Hence 


$$
v^{ \pm}(x)=u^{ \pm}(x) \text { for } \mathcal{H}^{n-1} \text { - a.e. } x \in \Gamma \backslash \bigcup_{i=1}^{N}\left(\partial^{*} \omega_{i}^{+} \cup \partial^{*} \omega_{i}^{-}\right)
$$

By the estimates of $\mathcal{H}^{n-1}\left(\partial^{*} \omega_{i}^{ \pm}\right)$at the end of Step 2 it follows that the traces of $u$ and $v$ on the two sides of $\Gamma$ can only differ on a small (in terms of $\alpha$ ) portion of $\Gamma$, namely

$$
\mathcal{H}^{n-1}\left(\Gamma \cap\left\{v^{ \pm} \neq u^{ \pm}\right\}\right) \leq 2 c \alpha \mathcal{H}^{n-1}(J)
$$

In conclusion, $w^{ \pm}=u^{ \pm}$in $\Gamma$, up to a set of small (in terms of $\alpha$ ) $\mathcal{H}^{n-1}$ measure. More precisely,

$$
w^{ \pm}(x)=u^{ \pm}(x) \text { for } \mathcal{H}^{n-1} \text { - a.e. } x \in \Gamma \backslash\left(\overline{\tilde{\omega}} \cup \hat{\omega}^{(1)} \cup \partial^{*} \hat{\omega}\right)
$$

and from (5.13) and (5.14)

$$
\mathcal{H}^{n-1}\left(\Gamma \cap\left\{w^{ \pm} \neq u^{ \pm}\right\}\right) \leq 3 c \alpha \mathcal{H}^{n-1}(J)
$$

Step 3.2: Estimate of $\hat{\omega}$. We now estimate the exceptional set $\hat{\omega}$, both in perimeter and in volume. Note that, by (5.11) and (5.8), $\mathcal{H}^{n-1}\left(\partial^{*} \omega_{G}\right) \leq C \sum_{q \in \mathcal{Q}_{g}} \mathcal{H}^{n-1}\left(J_{v} \cap q^{\prime \prime}\right) \leq$ $c \alpha\left(1+\mathcal{H}^{n-1}(J)\right)$, for some constant $c=c(n, p)$. We also remark that for each $q$, one has $\mathcal{L}^{n}\left(\omega_{q}\right) \leq c \delta \mathcal{H}^{n-1}\left(\partial^{*} \omega_{q}\right)$ for a dimensional constant $c$, so in particular

$$
\mathcal{L}^{n}\left(\omega_{G}\right) \leq c \delta \alpha\left(1+\mathcal{H}^{n-1}(J)\right)
$$

with $c=c(n, p)$.

Combining these estimates with the bound on $\mathcal{H}^{n-1}\left(\partial^{*} \omega_{B}\right)$ at the end of Step 2, we have that

$$
\mathcal{H}^{n-1}\left(\partial^{*} \hat{\omega}\right) \leq c \alpha\left(1+\mathcal{H}^{n-1}(J)\right)
$$

for a constant $c=c(n, p)$.

Step 3.3: $L^{p}$-estimate of $e(w)$. We start by estimating $\int_{\omega_{G}}|e(w)|^{p} d x$. From (5.12) we have, for $x \in G$,

$$
e(w)(x)=\sum_{q \in \mathcal{Q}_{g}}\left(e\left(w_{q}\right)(x) \varphi_{q}(x)+w_{q}(x) \odot \nabla \varphi_{q}(x)\right) .
$$

Note that, since the cubes $q^{\prime}$ have finite overlap, the sum in the right-hand side of (5.19) is done, at each point, over a uniformly bounded number of terms, depending on the dimension. 
We estimate the $L^{p}$ norm of the two terms of the sum in (5.19) separately. For the first term we have that

$$
\begin{aligned}
& \int_{\omega_{G}}\left|\sum_{q \in \mathcal{Q}_{g}} e\left(w_{q}\right)(x) \varphi_{q}(x)\right|^{p} d x \leq \sum_{\hat{q} \in \mathcal{Q}_{g}} \int_{\omega_{\hat{q}}}\left|\sum_{q \in \mathcal{Q}_{g}} e\left(w_{q}\right)(x) \varphi_{q}(x)\right|^{p} d x
\end{aligned}
$$

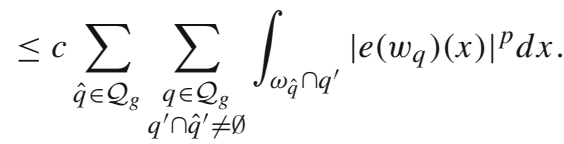

For fixed $\hat{q}$ and $q$ with $q^{\prime} \cap \hat{q}^{\prime} \neq \emptyset$ we estimate

$$
\begin{aligned}
\int_{\omega_{\hat{q}} \cap q^{\prime}}\left|e\left(w_{q}\right)(x)\right|^{p} d x & =\int_{\left(\omega_{\hat{q}} \cap \omega_{q}\right) \cap q^{\prime}}\left|e\left(w_{q}\right)(x)\right|^{p} d x+\int_{\left(\omega_{\hat{q}} \backslash \omega_{q}\right) \cap q^{\prime}}|e(v)(x)|^{p} d x \\
& \leq \int_{\omega_{q}}\left|e\left(w_{q}\right)(x)\right|^{p} d x+\int_{\omega_{\hat{q}}}|e(v)(x)|^{p} d x \\
& \leq 2 \int_{\omega_{q}}|e(v)(x)|^{p} d x+\int_{\omega_{\hat{q}}}|e(v)(x)|^{p} d x
\end{aligned}
$$

where in the last step we used (5.10). Since the cubes $q^{\prime}$ have finite overlap, from (5.20) we conclude that

$$
\int_{\omega_{G}}\left|\sum_{q \in \mathcal{Q}_{g}} e\left(w_{q}\right)(x) \varphi_{q}(x)\right|^{p} d x \leq c \int_{\omega_{G}}|e(v)|^{p} d x .
$$

Therefore,

$$
\begin{aligned}
\int_{\omega_{G}}\left|\sum_{q \in \mathcal{Q}_{g}} e\left(w_{q}\right)(x) \varphi_{q}(x)\right|^{p} d x & \leq c \int_{\omega_{G}}|e(v)|^{p} d x \\
& =c\left(\int_{\omega_{G} \backslash \cup_{i} B_{i}}|e(v)|^{p} d x+\sum_{i=1}^{N} \int_{\omega_{G} \cap B_{i}}|e(v)|^{p} d x\right) \\
& \leq c\left(\int_{\omega_{G}}|e(u)|^{p} d x+\sum_{i=1}^{N} \int_{B_{i}}|e(u)|^{p} d x\right),
\end{aligned}
$$

where in the last inequality we have used the definition of $v$, and in particular the fact that $v=u$ outside $\cup_{i} B_{i}$, and the estimate of $e\left(v_{i}^{ \pm}\right)$in terms of $e(u)$ (see Step 2).

We now estimate the second term of the sum in (5.19). For $x \in G$ we define $\mathcal{Q}_{g}^{x}:=\left\{q \in \mathcal{Q}_{g}: \varphi_{q}(x)>0\right\}$, and denote $N_{\mathcal{Q}}^{x}:=\# \mathcal{Q}_{g}^{x}$ (which, as already observed, is uniformly bounded by a quantity depending only on the dimension, namely $2^{n}$ ). 
Using that $\sum_{q \in \mathcal{Q}_{g}^{x}} \nabla \varphi_{q}(x)=0$, one has

$$
\begin{aligned}
\sum_{q \in \mathcal{Q}_{g}} w_{q}(x) \odot \nabla \varphi_{q}(x) & =\sum_{q \in \mathcal{Q}_{g}^{x}}\left(w_{q}(x)-\frac{1}{N_{\mathcal{Q}}^{x}} \sum_{\hat{q} \in \mathcal{Q}_{g}^{x}} w_{\hat{q}}(x)\right) \odot \nabla \varphi_{q}(x) \\
& =\frac{1}{N_{\mathcal{Q}}^{x}} \sum_{q, \hat{q} \in \mathcal{Q}_{g}^{x}}\left(w_{q}(x)-w_{\hat{q}}(x)\right) \odot \nabla \varphi_{q}(x) .
\end{aligned}
$$

Since $q, \hat{q} \in \mathcal{Q}_{g}^{x} \Rightarrow x \in q^{\prime} \cap \hat{q}^{\prime}$, to bound the $L^{p}$ norm of the above expression, it is enough to estimate

$$
\int_{q^{\prime} \cap \hat{q}^{\prime}}\left|w_{q}-w_{\hat{q}}\right|^{p}\left|\nabla \varphi_{q}\right|^{p} d x
$$

for any pair of neighbouring cubes $q, \hat{q} \in \mathcal{Q}_{g}^{x}$. Note that $w_{q}-w_{\hat{q}} \in W^{1, p}\left(q^{\prime} \cap \hat{q}^{\prime} ; \mathbb{R}^{n}\right)$ and $w_{q}-w_{\hat{q}}=0$ in $\left(q^{\prime} \cap \hat{q}^{\prime}\right) \backslash\left(\omega_{q} \cup \omega_{\hat{q}}\right)$, since both functions coincide with $v$. Moreover, since $\mathcal{L}^{n}\left(q^{\prime} \cap \hat{q}^{\prime}\right) \geq \delta^{n} / 8^{n}$ and $\mathcal{L}^{n}\left(\omega_{q} \cup \omega_{\hat{q}}\right) \leq C \tau^{n /(n-1)} \delta^{n}$ for some dimensional constant $C$, provided $\tau$ is chosen small enough one can ensure that

$$
\mathcal{L}^{n}\left(\left\{x \in q^{\prime} \cap \hat{q}^{\prime}: w_{q}-w_{\hat{q}}=0\right\}\right) \geq \frac{1}{2} \mathcal{L}^{n}\left(q^{\prime} \cap \hat{q}^{\prime}\right)
$$

One can then easily deduce from Lemma 4.4 that, for some constant $c$ (depending on $p$ and on the dimension):

$$
\int_{q^{\prime} \cap \hat{q}^{\prime}}\left|w_{q}-w_{\hat{q}}\right|^{p} d x \leq c \delta^{p} \int_{q^{\prime} \cap \hat{q}^{\prime}}\left|e\left(w_{q}-w_{\hat{q}}\right)\right|^{p} d x \leq c \delta^{p} \int_{\omega_{q} \cup \omega_{\hat{q}}}\left|e\left(w_{q}-w_{\hat{q}}\right)\right|^{p} d x
$$

Since $\left|\nabla \varphi_{q}\right| \leq C / \delta$ in each cube, we can estimate (5.22) as

$$
\begin{aligned}
\int_{q^{\prime} \cap \hat{q}^{\prime}}\left|w_{q}-w_{\hat{q}}\right|^{p}\left|\nabla \varphi_{q}\right|^{p} d x & \leq c \int_{\left(q^{\prime} \cap \hat{q}^{\prime}\right) \cap\left(\omega_{q} \cup \omega_{\hat{q}}\right)}\left|e\left(w_{q}-w_{\hat{q}}\right)\right|^{p} d x \\
& \leq c\left(\int_{q^{\prime} \cap\left(\omega_{q} \cup \omega_{\hat{q}}\right)}\left|e\left(w_{q}\right)\right|^{p} d x+\int_{\hat{q}^{\prime} \cap\left(\omega_{q} \cup \omega_{\hat{q}}\right)}\left|e\left(w_{\hat{q}}\right)\right|^{p} d x\right) \\
& \leq c \int_{\omega_{q} \cup \omega_{\hat{q}}}|e(v)|^{p} d x .
\end{aligned}
$$

Hence we have that

$$
\int_{\omega_{G}}\left|\sum_{q \in \mathcal{Q}_{g}} w_{q}(x) \odot \nabla \varphi_{q}(x)\right|^{p} d x \leq c \int_{\omega_{G}}|e(v)|^{p} d x
$$


which, together with (5.21), gives, from (5.19),

$$
\int_{\omega_{G}}|e(w)|^{p} d x \leq c \int_{\omega_{G} \cup\left(\bigcup_{i=1}^{N} B_{i}\right)}|e(u)|^{p} d x .
$$

Finally, we estimate $\int_{\mathbb{R}^{n} \backslash(\Gamma \cup \tilde{\omega})}|e(w)|^{p} d x$. We have

$$
\begin{aligned}
& \int_{\mathbb{R}^{n} \backslash(\Gamma \cup \tilde{\omega})}|e(w)|^{p} d x \leq \int_{\mathbb{R}^{n} \backslash(\Gamma \cup \tilde{\omega} \cup \hat{\omega})}|e(w)|^{p} d x+\int_{\left(\mathbb{R}^{n} \backslash(\Gamma \cup \tilde{\omega})\right) \cap \hat{\omega}}|e(w)|^{p} d x \\
& \leq \int_{\mathbb{R}^{n}}|e(u)|^{p} d x+\int_{\left(\mathbb{R}^{n} \backslash \tilde{\omega}\right) \cap \omega_{G}}|e(w)|^{p} d x+\int_{\left(\mathbb{R}^{n} \backslash\left(\tilde{\omega} \cup \omega_{G}\right)\right) \cap \omega_{B}}|e(w)|^{p} d x,
\end{aligned}
$$

since $w=u$ in $\mathbb{R}^{n} \backslash(\tilde{\omega} \cup \hat{\omega})$. Using that $w=v$ outside $\tilde{\omega} \cup \omega_{G}$ we have

$$
\int_{\left(\mathbb{R}^{n} \backslash\left(\tilde{\omega} \cup \omega_{G}\right)\right) \cap \omega_{B}}|e(w)|^{p} d x=\int_{\left(\mathbb{R}^{n} \backslash\left(\tilde{\omega} \cup \omega_{G}\right)\right) \cap \omega_{B}}|e(v)|^{p} d x \leq c \sum_{i=1}^{N} \int_{B_{i}}|e(u)|^{p} d x,
$$

where the last inequality follows from the definition of $\omega_{B}$, the fact that $v=v_{i}^{ \pm}$in $B_{i}^{ \pm}$, and the bound (5.5), for $i=1, \ldots, N$.

In conclusion, from (5.24), (5.23) and (5.25) it follows that

$$
\int_{\mathbb{R}^{n} \backslash(\Gamma \cup \tilde{\omega})}|e(w)|^{p} d x \leq \int_{\mathbb{R}^{n}}|e(u)|^{p} d x+c \int_{\omega_{G} \cup\left(\bigcup_{i=1}^{N} B_{i}\right)}|e(u)|^{p} d x .
$$

As a consequence, if we recall point e) of the construction of the $B_{i}$ 's and (5.17) above, if $\rho>0$ and $\delta>0$ are chosen small enough, one can ensure that

$$
\int_{\mathbb{R}^{n} \backslash(\Gamma \cup \tilde{\omega})}|e(w)|^{p} d x \leq(1+\alpha) \int_{\mathbb{R}^{n}}|e(u)|^{p} d x .
$$

(Note that for (5.26) to hold true, the choice of $\rho>0$ and $\delta>0$ makes them dependent on the function $u$, but $\alpha$ is independent of $u$.) By choosing $\alpha$ sufficiently small in (5.3), (5.9), (5.15), (5.16), (5.18) and (5.26) the conclusion follows. 
Acknowledgements The authors would like to thank the referee for carefully reading the manuscript and for providing several helpful suggestions. The authors acknowledge the hospitality and support of the INI under the Grant EP/R014604/1.

Data Availibility Statements Data sharing not applicable to this article as no datasets were generated or analysed during the current study.

\section{Declarations}

Conflict of interest On behalf of all authors, the corresponding author states that there is no conflict of interest.

Open Access This article is licensed under a Creative Commons Attribution 4.0 International License, which permits use, sharing, adaptation, distribution and reproduction in any medium or format, as long as you give appropriate credit to the original author(s) and the source, provide a link to the Creative Commons licence, and indicate if changes were made. The images or other third party material in this article are included in the article's Creative Commons licence, unless indicated otherwise in a credit line to the material. If material is not included in the article's Creative Commons licence and your intended use is not permitted by statutory regulation or exceeds the permitted use, you will need to obtain permission directly from the copyright holder. To view a copy of this licence, visit http://creativecommons.org/licenses/by/4.0/.

\section{References}

1. Adams, R.A., Fournier, J.J.F: Sobolev spaces. Pure and Applied Mathematics (Amsterdam), Second edition, Elsevier/Academic Press, Amsterdam, (2003)

2. Ambrosio, L., Fusco, N., Pallara, D.: Functions of bounded variations and free discontinuity problems. Clarendon Press, Oxford (2000)

3. Ambrosio, L., Coscia, A., Dal Maso, G.: Fine properties of functions with bounded deformation. Arch. Rat. Mech. Anal. 139(3), 201-238 (1997)

4. Babadjian, J.-F., Iurlano, F., Lemenant, A.: Partial regularity for the crack set minimizing the twodimensional Griffith energy. Accepted for publication. J. Eur. Math. Soc. arXiv:1905.10298

5. Bellettini, G., Coscia, A., Dal Maso, G.: Compactness and lower semicontinuity properties in $\operatorname{SBD}(\Omega)$. Math. Z. 228, 337-351 (1998)

6. Cagnetti, F., Chambolle, A., Perugini, M., Scardia, L.: An extension result for generalised special functions of bounded deformation. J. Convex Anal. 28/2 (2021)

7. Chambolle, A.: An approximation result for special functions with bounded deformation. J. Math. Pures Appl. 83, 929-954 (2004)

8. Chambolle, A., Conti, S., Francfort, G.: Approximation of a brittle fracture energy with a constraint of non-interpenetration. Arch. Rat. Mech. Anal. 228(3), 867-889 (2018)

9. Chambolle, A., Conti, S., Francfort, G.: Korn-Poincaré Inequalities for Functions with a Small Jump Set. Indiana Univ. Math. J. 65(4), 1373-1399 (2016)

10. Chambolle, A., Conti, S., Iurlano, F.: Approximation of functions with small jump sets and existence of strong minimizers of Griffith's energy. J. Math. Pures Appl. (9) 128, 119-139 (2019)

11. Chambolle, A., Crismale, V.: A density result in $G S B D^{p}$ with applications to the approximation of brittle fracture energies. Arch. Rat. Mech. Anal. 232, 1329-1378 (2019)

12. Chambolle, A., Crismale, V.: Compactness and lower semicontinuity in GSBD. J. Eur. Math. Soc. 23(3), 701-719 (2021)

13. Chambolle, A., Crismale, V.: Existence of strong solutions to the Dirichlet problem for the Griffith energy. Calc. Var. Partial Differ. Equ. 58(136) (2019)

14. Chambolle, A., Crismale, V.: Equilibrium configurations for nonhomogeneous linearly elastic materials with surface discontinuities. Preprint arXiv:2006.00480

15. Conti, S., Focardi, M., Iurlano, F.: Existence of strong minimizers for the Griffith static fracture model in dimension two. Ann. Inst. H. Poincaré Anal. Non Linéaire 36, 455-474 (2019)

16. Conti, S., Focardi, M., Iurlano, F.: Integral representation for functionals defined on $S B D^{p}$ in dimension 2. Arch. Rat. Mech. Anal. 223, 1337-1374 (2017) 
17. Conti, S., Focardi, M., Iurlano, F.: Which special functions of bounded deformation have bounded variation? Proc. R. Soc. Edinburgh Sect. A 148, 33-50 (2018)

18. Conti, S., Focardi, M., Iurlano, F.: Approximation of fracture energies with $p$-growth via piecewise affine finite elements. ESAIM Control Optim. Calc. Var. 25(34) (2019)

19. Crismale, V.: Density in $S B D$ and approximation of fracture energies. Atti Accad. Naz. Lincei Rend. Lincei Mat. Appl. 30, 533-542 (2019)

20. Crismale, V.: On the approximation of $S B D$ functions and some applications. SIAM J. Math. Anal. 51, 5011-5048 (2019)

21. Crismale, V., Friedrich, M., Solombrino, F.: Integral representation for energies in linear elasticity with surface discontinuities. Adv. Calc. Var. arXiv:2005.06866

22. Dal Maso, G.: Generalised functions of bounded deformation. J. Eur. Math. Soc. 15(5), 1943-1997 (2013)

23. De Giorgi, E., Carriero, M., Leaci, A.: Existence theorem for a minimum problem with free discontinuity set. Arch. Rat. Mech. Anal. 108, 195-218 (1989)

24. Evans, L.C., Gariepy, R.F: Measure theory and fine properties of functions. Studies in advanced mathematics. CRC Press, Boca Raton, FL (1992)

25. Federer, H.: Geometric measure theory. Springer, Berlin (1969)

26. Friedrich, M.: A Korn-Poincaré-type inequality for special functions of bounded deformation (2015). Preprint arXiv:1503.06755

27. Friedrich, M.: A Korn-type inequality in $S B D$ for functions with small jump sets. Math. Models Methods Appl. Sci. 27, 2461-2484 (2017)

28. Friedrich, M.: A Piecewise Korn Inequality in $S B D$ and Applications to Embedding and Density Results. SIAM J. Math. Anal. 50, 3842-3918 (2018)

29. Iurlano, F.: A density result for $G S B D$ and its applications to the approximation of brittle fracture energies. Calc. Var. Partial Differ. Equ. 15, 315-342 (2014)

30. Leoni, G.: A first course in Sobolev spaces. Graduate Studies in Mathematics. 105. American Mathematical Society (AMS), Providence, RI, (2009)

31. Nitsche, J.A.: On Korn's second inequality. RAIRO. Anal. Numérique 15(3), 237-248 (1981)

Publisher's Note Springer Nature remains neutral with regard to jurisdictional claims in published maps and institutional affiliations. 